Orignal Research Paper

\title{
Modeling Hepatocellular Toxicity of Enavatuzumab, a Humanized Anti-TweakR Antibody
}

\author{
${ }^{1}$ Donghee Choi, ${ }^{2}$ Yanhong Zhu, ${ }^{3}$ Debra Tien Chao, ${ }^{3}$ Mien Sho, ${ }^{3}$ Susan Rhodes, \\ ${ }^{3}$ Melvin Fox, ${ }^{4}$ Gary Charles Starling and ${ }^{5}$ Patricia Ann Culp \\ ${ }^{1}$ Department of Oncology Discovery, AbbVie Biotherapeutics, 1500 Seaport Blvd, Redwood City, CA USA \\ ${ }^{2}$ Department of Toxicology, Dermira Inc., Menlo Park, CA USA \\ ${ }^{3}$ Department of Oncology Discovery, AbbVie Biotherapeutics, 1500 Seaport Blvd, Redwood City, CA USA \\ ${ }^{4}$ Department of Biologics \& Vaccines Discovery, Merck, USA \\ ${ }^{5}$ Department of Immuno-Oncology, Alector, USA
}

Corresponding Author:

Donghee Choi

Department of Oncology

Discovery, AbbVie

Biotherapeutics, 1500 Seaport

Blvd, Redwood City, CA USA

Tel: $650-454-2111$

Fax: 650-454-1477

Email: donghee.choi@abbvie.com

\begin{abstract}
In a Phase 1 clinical study, treatment with enavatuzumab, a humanized monoclonal antibody to TweakR, resulted in liver toxicity in a subset of patients. The objective of this current study was to evaluate the ability of preclinical studies to predict liver toxicity in humans. Enavatuzumab was evaluated in cynomolgus monkeys, where serum liver enzyme and cytokine levels were measured and histopathology of the liver was performed. TweakR expression was evaluated by immunohistochemistry in healthy human liver and in liver tissues from cancer patients. Enavatuzumab was also evaluated in vitro for its impact on human hepatocytes when cultured both alone and with immune cells. Enavatuzumab-treated cynomolgus monkeys exhibited liver enzyme elevations only at the highest dose level $(100 \mathrm{mg} / \mathrm{kg})$ and few cytokines were elevated after dosing. Bile duct hyperplasia was observed in the liver but appeared to be partially reversible. Compared with healthy liver, liver tissues from cancer patients exhibited marked elevation of TweakR expression, which was associated with immune cell infiltration. Enavatuzumab treatment of cultured hepatocytes, both in the presence and absence of immune cells resulted in increased cytokine release, but only in the co-cultures were liver enzymes elevated. These results suggest that due to differences in liver architecture between healthy humans and cancer patients, studies in healthy non-human primates may underestimate the potential for liver toxicity in human cancer patients. The use of additional in vitro assays in conjunction with in vivo studies may better predict the potential impact of a therapeutic agent on the liver in clinical studies.
\end{abstract}

Keywords: TweakR, Enavatuzumab, Liver Toxicity, Cytokine Release, Cynomolgus

\section{Introduction}

Liver toxicity is one of the most common adverse events observed after treatment with pharmaceutical agents (Zimmerman, 2000; Kaplowitz, 2004). The proposed mechanism for this exquisite sensitivity of the liver to small molecule drugs is the accumulation of compounds and their metabolites due to the role of the liver in metabolism and clearance.

A wide range of small molecule chemotherapeutics for oncology treatment, including targeted agents, induce liver toxicity in patients, with varying rates of incidence (Field et al., 2008; Field and Michael, 2008). Biologic agents, including antibodies, can also induce liver toxicity, although less prevalently than observed for small molecule therapies. The nature of liver toxicity with biologics can vary widely, from asymptomatic elevations of liver enzymes to fatal liver failure (Ogasawara et al., 1993; Vonderheide et al., 2007; Advani et al., 2009; Tolcher et al., 2007; Plummer et al., 2007). For many such agents that induce liver toxicity, the mechanisms of toxicity are often not well-understood.

Enavatuzumab is a humanized monoclonal antibody targeting TweakR (Fn14, TWEAK receptor, TNFRSF12A) that has been developed for the treatment of patients with solid tumors. 
Enavatuzumab induces potent anti-tumor activity in preclinical models through both directly inhibiting the growth of tumor cells and through Antibody-Dependent Cellular Cytotoxicity (ADCC) (Chao et al., 2013; Culp et al., 2010; Purcell et al., 2014). In a Phase 1 study in cancer patients, enavatuzumab treatment every two weeks induced asymptomatic elevations in ALT and AST at all dose levels tested $(0.1-1.5 \mathrm{mg} / \mathrm{kg})$ and the Maximum Tolerated Dose (MTD) was found to be $1 \mathrm{mg} / \mathrm{kg}$ (Lam et al., 2017). Both the frequency and magnitude of liver enzyme elevations increased with antibody dose level, suggesting a dose-response relationship.

In this study, we describe preclinical studies conducted with enavatuzumab, focusing on predicting the potential for liver toxicity in humans. In cynomolgus monkeys, enavatuzumab induced ALT elevations only at the highest dose level tested $(100 \mathrm{mg} / \mathrm{kg})$, indicating a disparity in the sensitivities of human cancer patients and non-human primates to enavatuzumab. In contrast to healthy human and cynomolgus liver tissues, liver samples from cancer patients were found to exhibit both inflammation and elevated TweakR expression. Treatment of normal hepatocytes in vitro with enavatuzumab induced release of cytokines, but not liver enzymes. However, in a co-culture of hepatocytes and immune cells, enavatuzumab induced both cytokine and ALT/AST release, both of which could be at least partially suppressed by pre-treatment with dexamethasone. Finally, dexamethasone pretreatment did not inhibit the anti-tumor activity of enavatuzumab in xenograft models, suggesting that dexamethasone pretreatment may be a means to suppress liver toxicity without compromising the antitumor activity of the antibody.

\section{Materials and Methods}

\section{Cells and Reagents}

Human lung fibroblasts were purchased from Lonza (Basel, Switzerland). Normal human hepatocytes were purchased from Lonza as fresh cultures in Hepatocyte Complete Media containing hydrocortisone. After receipt, the cells were maintained in Hepatocyte Complete Media without hydrocortisone for 1-3 days before using the cells in assays.

A freshly isolated lung from a cynomolgus monkey (Macaca fascicularis) was obtained from Charles River Laboratories (Wilmington, MA). The tissue was finely minced then treated with collagenase I for $6 \mathrm{~h}$ at room temperature. Tissue and dissociated cells were then washed several times with media and plated in the presence of $10 \mathrm{ng} / \mathrm{mL} \mathrm{bFGF}$ and $20 \mathrm{U} / \mathrm{mL}$ heparin. Six days later, fibroblasts that had grown out of the tissue were collected and stored at $-80^{\circ} \mathrm{C}$ for future use.

Peripheral Blood Mononuclear Cells (PBMCs) were isolated from whole blood collected from healthy human donors at AbbVie Biotherapeutics under an IRBapproved protocol. All experiments using PBMCs were performed with at least two different donors.

Enavatuzumab is a human IgG1 antibody to TweakR described previously (Culp et al., 2010). The isotype control antibody was MSL109 (Drobyski et al., 1991). Where indicated, antibodies were cross linked with a goat anti-human Fc $\gamma$ secondary antibody (Jackson Immunoresearch, West Grove, PA).

\section{Immunohistochemistry}

Frozen normal human tissues were collected from autopsy samples by Zoion Diagnostic (Shrewsbury, MA) with appropriate IRB approval. Frozen normal cynomolgus tissues were provided by Covance (Madison, WI). Frozen tissues were embedded in OCT medium and cut into $5 \mu \mathrm{m}$ sections onto slides with a Cryostat (Leica). Enavatuzumab was pre-incubated with a biotinylated secondary antibody for $30 \mathrm{~min}$ at room temperature. Human IgG was added to the complex solution immediately prior to incubation with tissue slides to minimize background staining. VECTASTAIN ABC Elite kit (Vector Labs, Burlingame, CA) was used to detect the signal and Hematoxylin Gill\#1 (Sigma. St. Louis, MO) was used for counterstaining.

Formalin-Fixed Paraffin Embedded (FFPE) liver biopsies were obtained from three cancer patients in the enavatuzumab Phase 1 study, Subjects 1102, 1111 and 1116 (Lam et al., 2017). Two additional FFPE liver samples from patients with cancer were obtained from Discovery Life Sciences (Los Osos, CA). Four of the five cancer patients had previously received chemotherapy treatment. All FFPE samples were stained for TweakR by immunohistochemistry as previously described (Culp et al., 2010).

\section{Antibody Treatment of Human and Cynomolgus Lung Fibroblasts}

A six point 3-fold serial dilution of enavatuzumab or a human IgG1 isotype control antibody was made in PBS for concentration starting from $300 \mu \mathrm{g} / \mathrm{mL}$ to $1 \mu \mathrm{g} / \mathrm{mL} .100 \mu \mathrm{L}$ of antibodies was added to an ELISA plate and incubated overnight at $4{ }^{\circ} \mathrm{C}$. The next day, the antibodies were removed and normal human or cynomolgus lung fibroblasts were added. After an overnight incubation at $37^{\circ} \mathrm{C}$, the cell supernatants were collected.

Cytokine and chemokine levels were quantified in supernatants by Luminex ${ }^{\circledR}$ using the Beadlyte ${ }^{\circledR}$ Human Multi-Cytokine Beadmaster ${ }^{\mathrm{TM}}$ Kit (Millipore, Billerica, MA). Supernatant $(50 \mu \mathrm{L})$ was incubated with Beadlyte ${ }^{\circledR}$ Human 22-plex Multi-Cytokine beads, according to the manufacturer's instructions. All samples and standards were assayed in duplicate. Of the 22 cytokines tested, only IL- 6 , IL- 8 , MCP-1, MIP$1 \alpha$ and RANTES were detected and the Luminex ${ }^{\circledR}$ 
reagents for these five cytokines cross-reacted efficiently with cynomolgus (Giavedoni, 2005).

\section{Toxicity Studies}

\section{One-Month Tolerability Study}

Purpose-bred cynomolgus monkeys at SNBL USA were administered enavatuzumab via a 30 -min intravenous (IV) infusion once weekly for 5 weeks. Two animals (one of each gender) were dosed at each of 4 dose levels: 0 (vehicle), 10,30 , or $100 \mathrm{mg} / \mathrm{kg}$.

Serum samples were collected pre-dose and at 1, 2, 6 and $24 \mathrm{~h}$ after the first dose for cytokine measurements. Serum samples were collected for serum biochemistry pre-dose and on D23 (24 h after the 4th dose) during the dosing period. At the conclusion of the study, animals were euthanized and a histopathology evaluation was performed at necropsy by a board-certified veterinarian pathologist.

IL-8, IL-6, GM-CSF, MCP-1, TNF $\alpha$ and IFN $\gamma$ were quantified in cynomolgus serum samples by Luminex ${ }^{\circledR}$, according to the manufacturer's instructions (Upstate, Temecula, CA). For most of the cytokines, $50 \mu \mathrm{L}$ of serum, diluted 1:1 with Assay Buffer, was analyzed. Quantification of RANTES was performed similarly, except that the serum was diluted 1:500 with Assay Buffer. All samples and standards were assayed in duplicate.

The tolerability study was conducted under the guidelines of the SNBL Institutional Animal Care and Use Committee (IACUC). The study was not conducted in compliance with the FDA Good Laboratory Practice (GLP) Regulations for Nonclinical Laboratory Studies.

\section{3-Week Toxicity Study}

Twenty one naïve male and 21 naïve female purposebred cynomolgus monkeys were treated via a $30 \mathrm{~min}$ intravenous (IV) infusion once every other week for 13 weeks, for a total of 7 doses, at $0,3,10,30$, or $100 \mathrm{mg} / \mathrm{kg}$. The distribution of animals among the groups is shown in Supplemental Table S1. Dosing and recovery phases began on days designated D1 and R1, respectively. Subsequent days in each phase were numbered incrementally. Animals in the Main Study group were sacrificed on D92, 7 days after the 7 th dose. For the animals in the Recovery group, a 24-week recovery period (R1-R170) followed administration of the last dose.
Liver enzymes were measured pre-dose, on D37 (8 days after the 3rd dose), D92 and at five time points during the recovery period: R41, R57, R85, R113, R141 and $\mathrm{R} 170$. Of the recovery time points, only results at R41 are shown, as no liver enzyme levels were elevated after that time point. Histopathology was conducted on tissues collected at necropsy in both the Main and Recovery groups.

The 13-week toxicity study was conducted at SNBL USA under the guidelines of their IACUC and in full compliance with the Food and Drug Administration GLP regulations for Nonclinical Laboratory Studies (21 CFR, Part 58).

\section{Flow Cytometry}

Human and cynomolgus hepatocytes were purchased from BD Biosciences (San Jose, CA). Human and cynomolgus hepatocytes or lung fibroblasts were incubated with primary antibody (enavatuzumab or isotype control antibody) in $50 \mu \mathrm{L}$. After washing away unbound primary antibody, cells were incubated with a fluorescein-conjugated goat anti-human IgG antibody (Caltag). Samples were analyzed on a FACScan ${ }^{\mathrm{TM}}$ by flow cytometry.

\section{Xenograft Studies}

NCI-H358 lung cancer cells were purchased from American Type Culture Collection and the SN12C cell line was obtained from Developmental Therapeutics Program/Division of Cancer Treatment and Diagnosis Tumor Repository at the National Cancer Institute. All cells were cultured as recommended by the supplier.

Human tumor cells, suspended in RPMI, were inoculated subcutaneously into the right flank of 6-week old ICR-SCID mice at $1 \times 10^{7}$ cells per mouse. When the tumors reached approximately $100 \mathrm{~mm}^{3}$ (length $\times$ width $\times$ height $/ 2$ ), the animals were randomized into dosing groups. Antibodies were administered at $1 \mathrm{mg} / \mathrm{kg}$, thrice per week and dexamethasone was administered at 5 $\mathrm{mg} / \mathrm{kg}$, with the initial dose given $24 \mathrm{~h}$ prior to the first antibody dose and subsequent doses given on alternate days to antibody dosing. All animal work was carried out under the NIH guidelines "Guide for the Care and Use of Laboratory Animals" following protocols approved by the AbbVie Biotherapeutics Institutional Animal Care and Use Committee.

Table S1: Dosing groups in the 13-week toxicity study

\begin{tabular}{llll}
\hline Table S1: Dosing groups in the 13-week toxicity study & Necropsy (male/female) \\
$\begin{array}{l}\text { Dose level } \\
(\mathrm{mg} / \mathrm{kg})\end{array}$ & $\begin{array}{l}\text { Number of } \\
\text { Animals (male/female) }\end{array}$ & Main (D92) & Recovery (R170) \\
\hline 0 & $5 / 5$ & $3 / 3$ & $2 / 2$ \\
3 & $3 / 3$ & $3 / 3$ & $2 / 0$ \\
10 & $5 / 5$ & $3 / 3$ & $0 / 0$ \\
30 & $3 / 3$ & $3 / 3$ & $2 / 2$ \\
100 & $5 / 5$ & $3 / 3$ & \\
\hline
\end{tabular}




\section{Sequencing of Cynomolgus Monkey TweakR}

Cynomolgus TweakR was amplified from 5' RACE products of cynomogus tissues using PCR primers designed to the published human TweakR/Fn14 sequence (NM_016639.1). The N-terminal portion of cynomolgus TweakR was isolated from cynomolgus PBMC, liver, thymus and thyroid RACE products using the following primers:

\section{Forward 5' GGCGCAGGACGTGCA CTATG Reverse 5' AGCGGCCGCTGCTGCAGCGCAGCC CAGGC}

The C-terminal portion of cynomolgus TweakR was amplified by PCR from a mixture of cynomolgus PBMC, liver, stomach, cerebral cortex and spleen RACE products using the following primers:

\section{Forward5' ATTGGATCCTGGAGACGATGCCGC AGGAG}

\section{Reverse 5' GTCTGGGAGGCAGAGACTGGC}

The internal portion of cynomolgus TweakR was amplified by PCR from the cynomolgus kidney RACE product using the following primers:

\section{Forward 5' TTCCTGGAGCGCGGACCTGG Reverse 5' GCCGGTCTCCTCTATGG}

A second internal fragment of cynomolgus TweakR was amplified from the cynomolgus spleen RACE product using the following primers:

\section{Forward 5' TCCGTGGCTGGGGAGCAAGC Reverse 5' GCCGGTCTCCTCTATGG}

All PCR products were sub cloned into the TOPA ${ }^{\circledR}$ TA cloning vector (Invitrogen). Multiple clones containing inserts of the appropriate size were sequenced.

\section{Hepatocyte and PBMC Assays}

Normal human hepatocytes were maintained in steroidfree media for $24 \mathrm{~h}$. Enavatuzumab or isotype control antibody $(10 \mu \mathrm{g} / \mathrm{mL})+/$-anti-human secondary antibody $(3.3 \mu \mathrm{g} / \mathrm{mL})$ was added and incubated for an additional 24 $\mathrm{h}$, after which the supernatants were collected.

PBMCs were cultured in the presence of enavatuzumab or a human IgG1 isotype control antibody $(10 \mu \mathrm{g} / \mathrm{mL}) .24 \mathrm{~h}$ later, the plates were centrifuged and supernatants were collected.

Co-culture assay: Normal human hepatocytes in 48-well plates (approximately $2 \times 10^{5}$ cells/well) were maintained in steroid-free media for $24 \mathrm{~h}$, after which freshly-isolated PBMCs $\left(2 \times 10^{6}\right.$ cells/well) were added. Dexamethasone (10 $\mu \mathrm{M}$ ) was added to some wells and $24 \mathrm{~h}$ later, enavatuzumab or isotype control antibody $(10 \mu \mathrm{g} / \mathrm{mL})$ was added. The supernatants were collected $24 \mathrm{~h}$ later.
Transwell $^{\circledR}$ assay: Normal human hepatocytes were maintained in steroid-free media for $3 \mathrm{~d}$, after which $10 \mu \mathrm{M}$ dexamethasone was added to some wells for 24 $\mathrm{h}$ prior to addition of the Transwell ${ }^{\circledR}$ containing PBMCs. PBMCs were maintained at $37^{\circ} \mathrm{C}$ for $24 \mathrm{~h}$ after isolation in the presence or absence of $10 \mu \mathrm{M}$ dexamethasone before adding to the Transwell ${ }^{\circledR}$. After 4 and $24 \mathrm{~h}$, the number of migrated PBMCs was enumerated by collecting cells from the bottom chamber, staining for CD45 and quantifying with Fluoresbrite Calibration Beads (Polysciences, Warrington, PA) by flow cytometry.

ALT and AST levels were measured in supernatants from the bottom chamber using Max Discovery ${ }^{\mathrm{TM}}$ Alanine Transaminase (ALT) Color Endpoint Assay Kit and Max Discovery ${ }^{\mathrm{TM}}$ Aspartate Transaminase (AST) Cytotoxicity Assay Kit purchased from Bioo Scientific Corporation (Austin, TX).

Cytokine and chemokine levels were quantified in supernatants from the bottom chamber by Luminex ${ }^{\circledR}$ using the Milliplex MAP Human Cytokine/Chemokine Kit (Millipore). Supernatant $(50 \mu \mathrm{L})$ was incubated with Beadlyte $^{\circledR}$ Human 26-plex Multi-Cytokine beads, according to the manufacturer's instructions. All samples and standards were assayed in duplicate.

\section{Results}

\section{Enavatuzumab Exhibited Similar Binding and Functional Activity on Human and Cynomolgus Cells}

Cynomolgus was chosen as the species in which to perform toxicity studies for enavatuzumab because the amino acid sequences of TweakR in human and cynomologus were found to be identical and enavatuzumab exhibited similar binding to normal tissues derived from the two species by immunohistochemistry and flow cytometry (Supplemental Fig. S1 and Fig. 1A). As enavatuzumab is a TweakR agonist antibody and cytokine release is a common downstream effect of activating the TweakR pathway (Burkly et al., 2011; Campbell et al., 2004), the ability of enavatuzumab to stimulate cytokine release from TweakR-expressing cultured normal human and cynomolgus cells was assessed. Enavatuzumab treatment of human or cynomolgus lung fibroblasts resulted in increased IL-8 levels from cells derived from both species, but IL-6 release only from human cells (Fig. 1B). Other cytokines and chemokines, including MCP-1, MIP- $1 \alpha$ and RANTES, were detected in supernatants of lung fibroblasts from both species but were not elevated by enavatuzumab in either species (data not shown). 


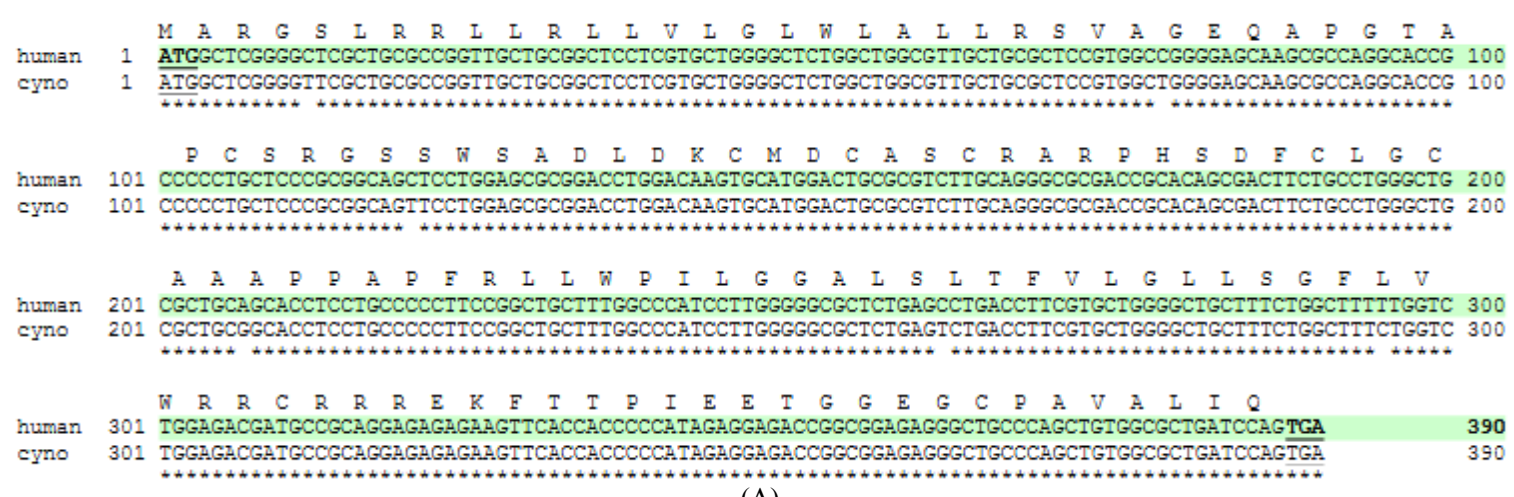

(A)

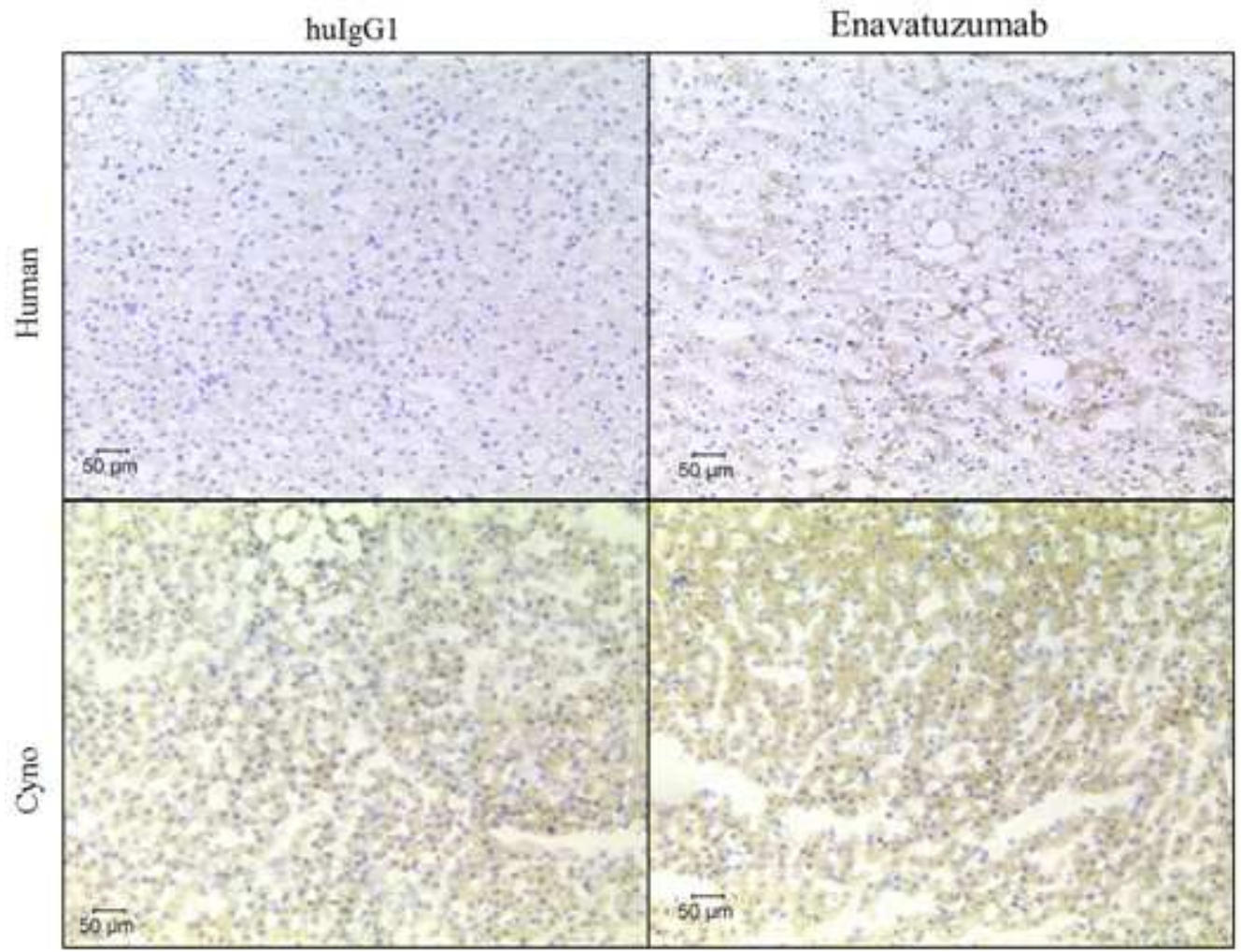

(B)

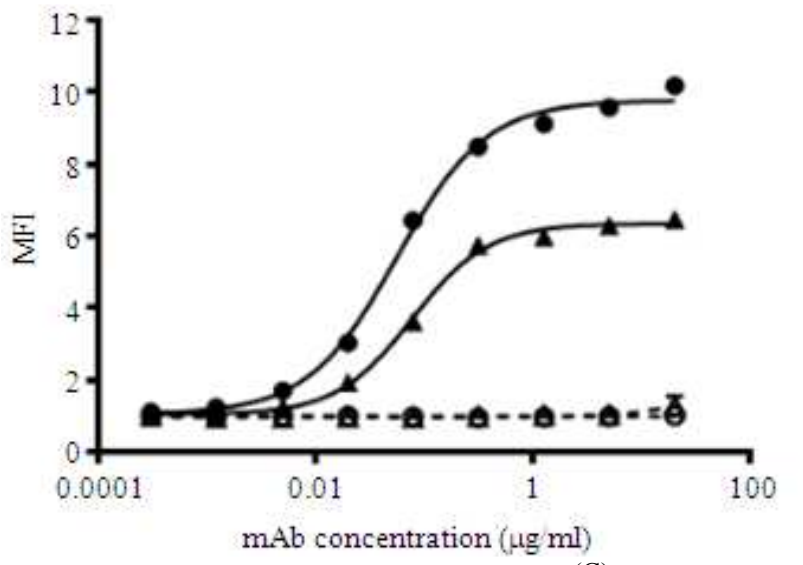

- Cyno enavatuzumab

A Human enavatuzumab

O Cyno hulgG1

$\Delta$ Human hulgG1 

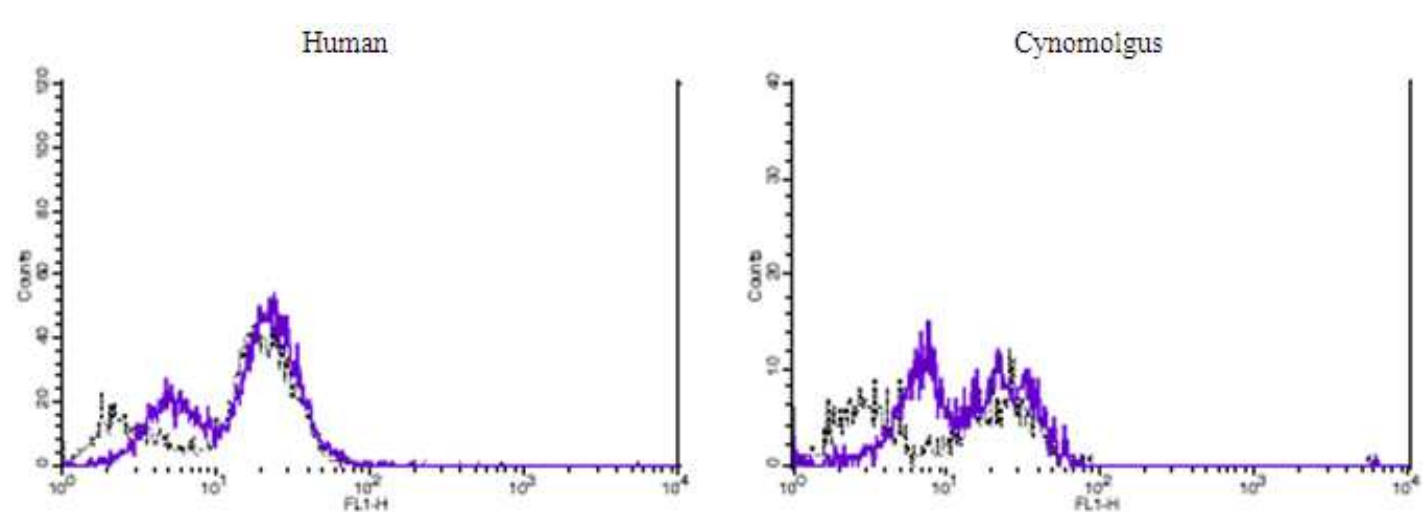

(D)

Fig. S1: Enavatuzumab binding and activity on normal tissues is similar in human and cynomolgus (A) Alignment of the published human (NM_016639.1) and PCR amplified cynomolgus TweakR nucleotide sequences and translated protein sequence (top line). Human and cynomolgus TweakR sequences differ at 6 nucleotides, but upon translation these changes do not lead to any differences at the amino acid level. The start (ATG) and stop (TGA) codons are underlined. * indicates human and cynomolgus TweakR identical nucleotide sequences, (B) Frozen liver tissues from healthy human and cynomolgus donors were stained with enavatuzumab or an isotype control antibody (huIgG1) by immunohistochemistry. Weak nonspecific staining was observed with both the isotype control antibody and enavatuzumab on human and cynomolgus liver tissues. The representative images are shown in 200X magnification (C) Binding of enavatuzumab and an isotype control antibody to normal human and cynomolgus lung fibroblasts was assessed by flow cytometry. Mean Fluorescence Intensity (MFI) is indicated on the Y-axis. Enavatuzumab bound human and cynomolgus cells with similar EC 50 (0.076 and $.055 \mu \mathrm{g} / \mathrm{mL}$, respectively). (D) Enavatuzumab (purple) and an isotype control antibody (black) at $10 \mu \mathrm{g} / \mathrm{mL}$ were incubated with normal human and cynomolgus hepatocytes, after which antibody binding was detected by flow cytometry. Both human and cynomolgus hepatocytes contained two populations of cells, one of which was stained by enavatuzumab in both species

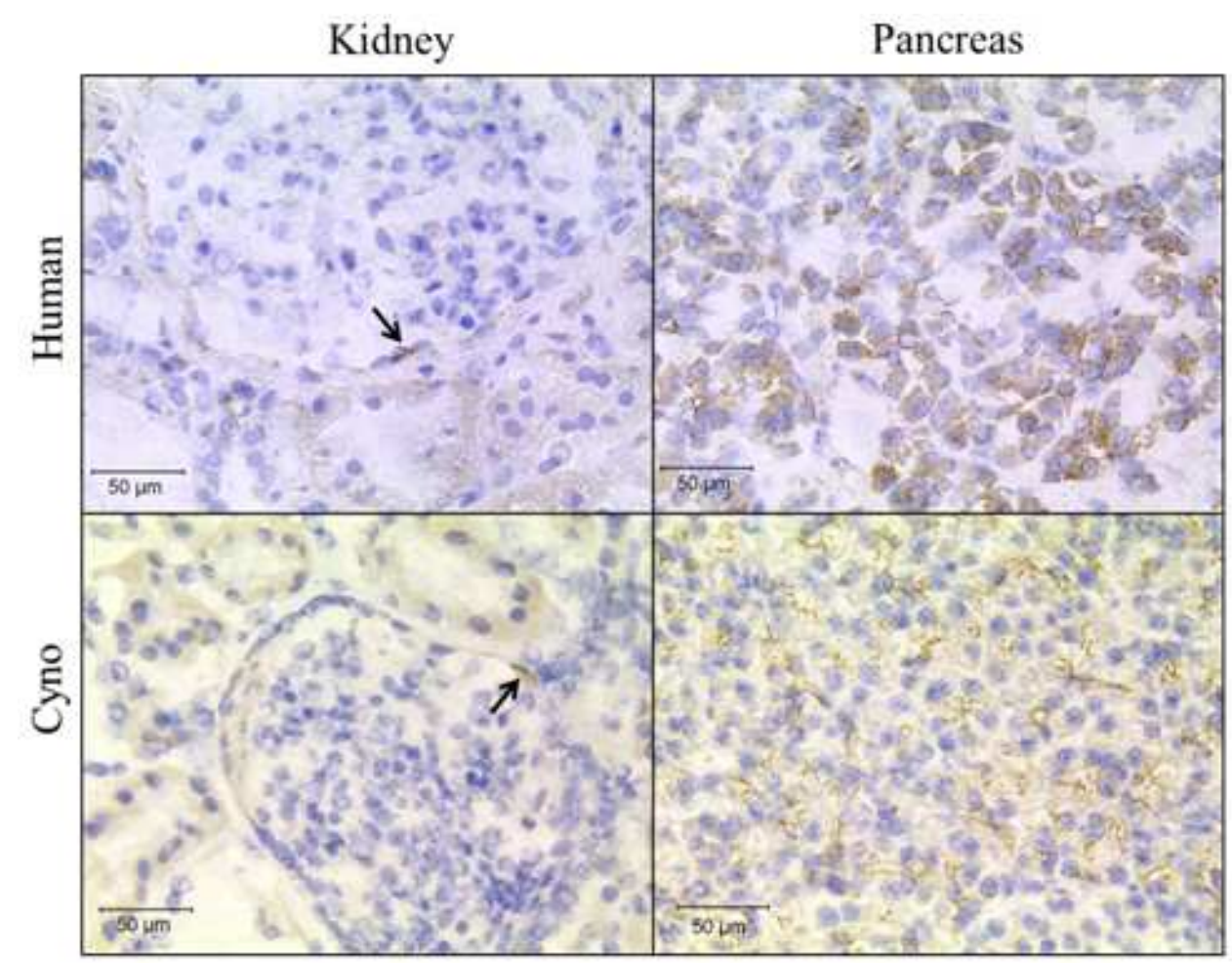

(A) 
IL-8
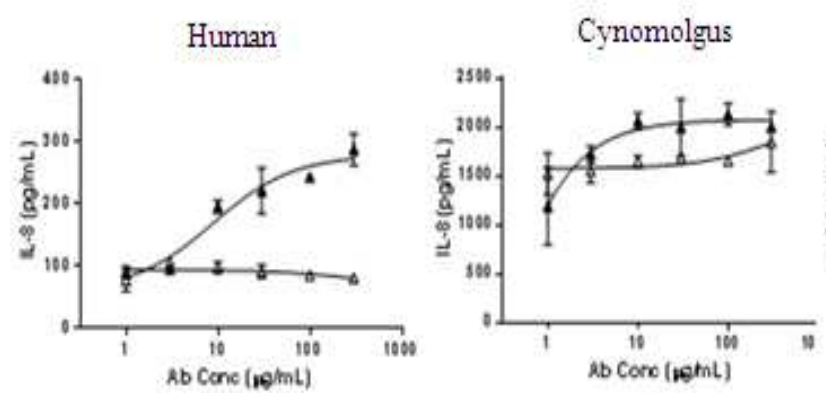

IL-6

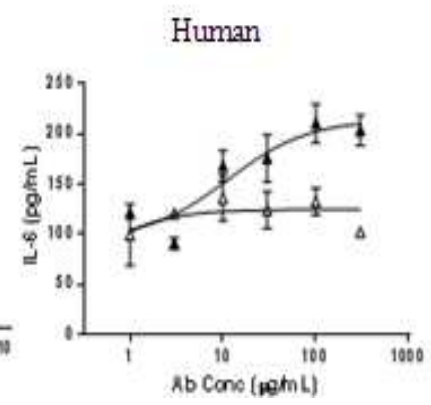

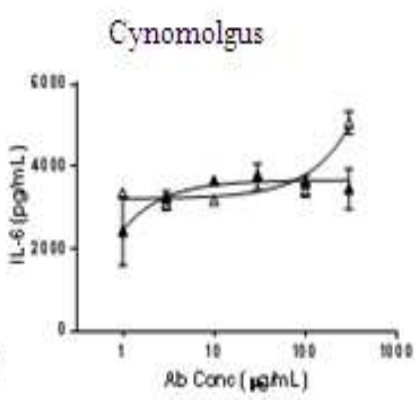

(B)

Fig. 1: Enavatuzumab binding and activity on normal tissues is similar in human and cynomolgus (A) Frozen kidney and pancreas tissues from healthy human and cynomolgus donors were stained with enavatuzumab by immunohistochemistry. Enavatuzumab stained the Bowman's capsule of the glomeruli in the kidney (arrow) in both species and exhibited membranous staining on human and cynomolgus pancreas samples. The representative images are shown in 600x magnification, (B) Cynomolgus and human lung fibroblasts were incubated with immobilized enavatuzumab (closed symbol) or an isotype control antibody (open symbol) for $24 \mathrm{~h}$, after which cytokines and chemokines were measured in the supernatants

\section{Enavatuzumab was Well-Tolerated in Non- Human Primates}

To evaluate the impact of enavatuzumab in vivo, two toxicity studies were performed in cynomolgus monkeys, a one-month tolerability study and a 13week GLP toxicity study. In the tolerability study, animals were administered enavatuzumab at one-week intervals, receiving five doses total. As cytokine release has been well-established as a downstream effect of TweakR activation, levels of circulating cytokines were measured in serum from the monkeys after the first dose of enavatuzumab. IL-6 appeared to be elevated in response to enavatuzumab treatment, but only in the mid and high dosing groups, where one animal in each of the $30 \mathrm{mg} / \mathrm{kg}$ and $100 \mathrm{mg} / \mathrm{kg}$ dosing groups exhibited post-dose IL-6 levels significantly above that in vehicle-dosed animals (Fig. 2A). In contrast, RANTES levels were elevated in post-dose samples in all dosing groups, including the vehicledosed group, suggesting that the RANTES elevations were not related to enavatuzumab administration. Other cytokines tested were either not elevated after enavatuzumab treatment or were not detected in any samples (data not shown).

Serum biochemistry was also analyzed in the tolerability study. Compared to pre-dose levels, ALT increased modestly in monkeys dosed with $30 \mathrm{mg} / \mathrm{kg}$, while AST was increased in animals in both the 10 $\mathrm{mg} / \mathrm{kg}$ and $30 \mathrm{mg} / \mathrm{kg}$ dosing groups (Fig. 2B). Only the increases in the $30 \mathrm{mg} / \mathrm{kg}$ group were significantly different from the control group and these elevations were within the normal limits. Other serum measurements of liver function, including alkaline phosphatase, bilirubin and albumin levels, showed no indication of liver toxicity induced by enavatuzumab (Supplemental Fig. S2). Histopathology performed on liver samples at the end of the study revealed no changes considered related to enavatuzumab.

In a second toxicity study in cynomolgus monkeys, a 13-week GLP toxicity study, animals were administered enavatuzumab once every two weeks, receiving a total of seven doses. As in the tolerability study, measurements of liver function were largely within the normal range (Fig. 2C). The exception was in the $100 \mathrm{mg} / \mathrm{kg}$ dosing group where ALT elevations were observed in two animals on D92 during the dosing period, with elevations of $2.4 \mathrm{x}$ and $3.4 \mathrm{x}$ over baseline in the two animals. These two animals were in the Main Study group and sacrificed at the end of the dosing period; thus ALT recovery in these animals was not able to be monitored. Also observed in the $100 \mathrm{mg} / \mathrm{kg}$ dosing group were minimal decreases in albumin levels in 3 of 5 male animals and 1 of 5 females. Modest AST increases were observed in two animals in the $10 \mathrm{mg} / \mathrm{kg}$ dosing group, but no doseresponse relationship was observed. ALP and bilirubin levels were not altered in any animals (Supplemental Fig. S2).

In the 13-week toxicity study, the animals in the Main Study group were sacrificed at the end of the dosing period (Day 92), with the remaining animals sacrificed 24 weeks later (R170), having received no additional treatment. From histopathology analysis on the liver tissues, bile duct hyperplasia was noted at necropsy of the Main Study group (Table 1). 

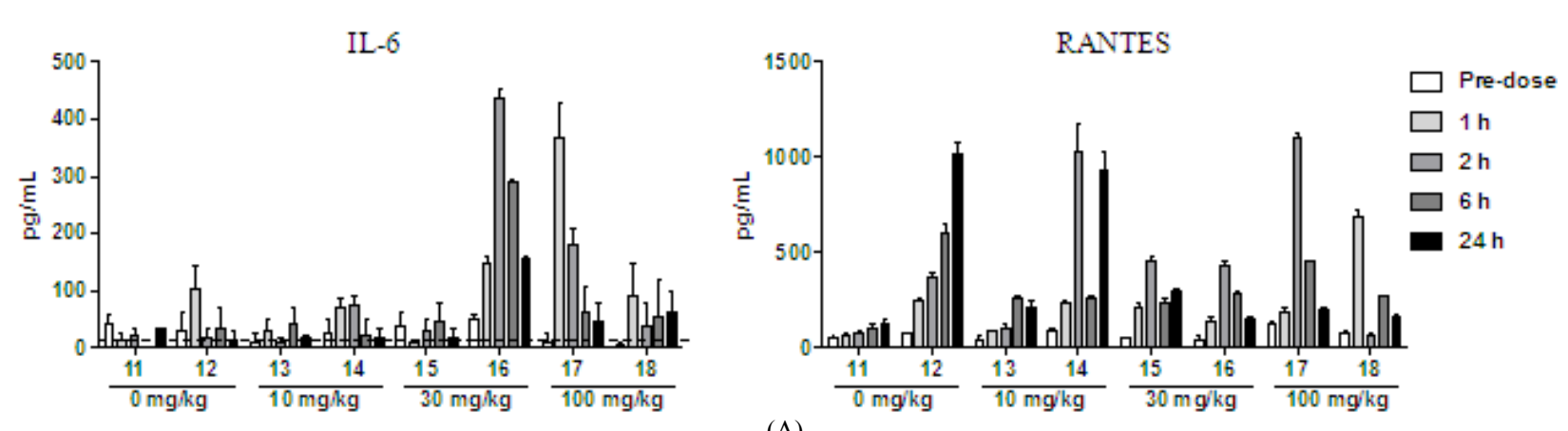

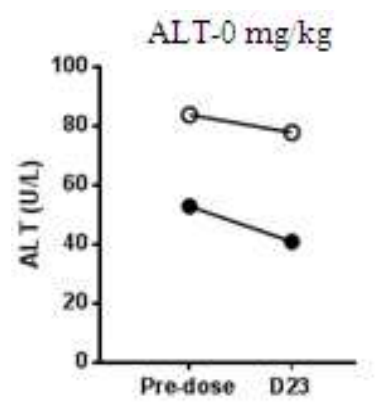

AST-0 mg kg

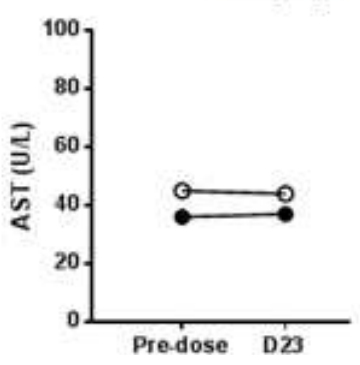

ALT-0 $\mathrm{mg} \mathrm{kg}$

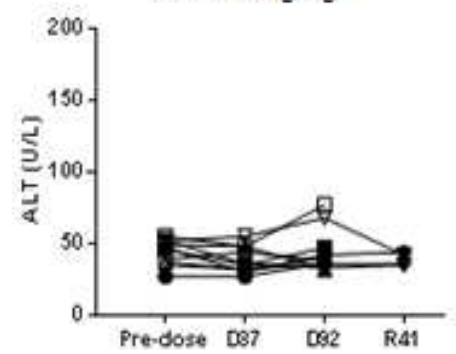

ALT-100 mg kg

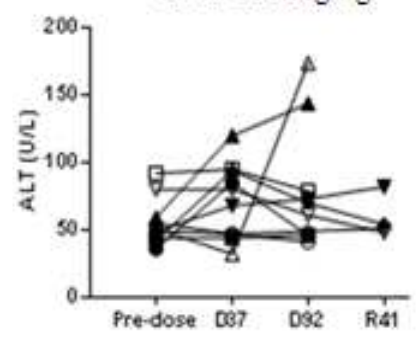

AIT-10 $\mathrm{mg} \mathrm{kg}$

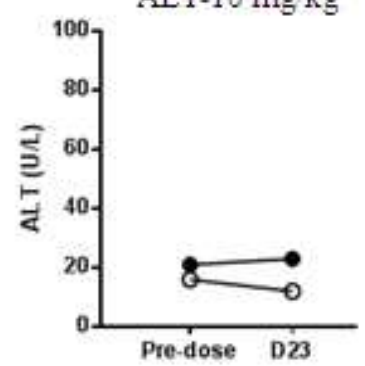

AST-10 mg kg

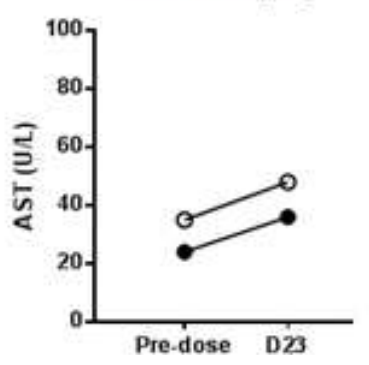

(B)

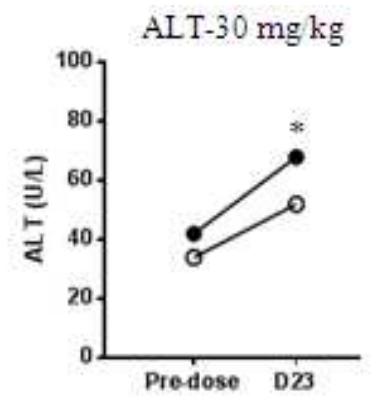

AST-30 mg kg
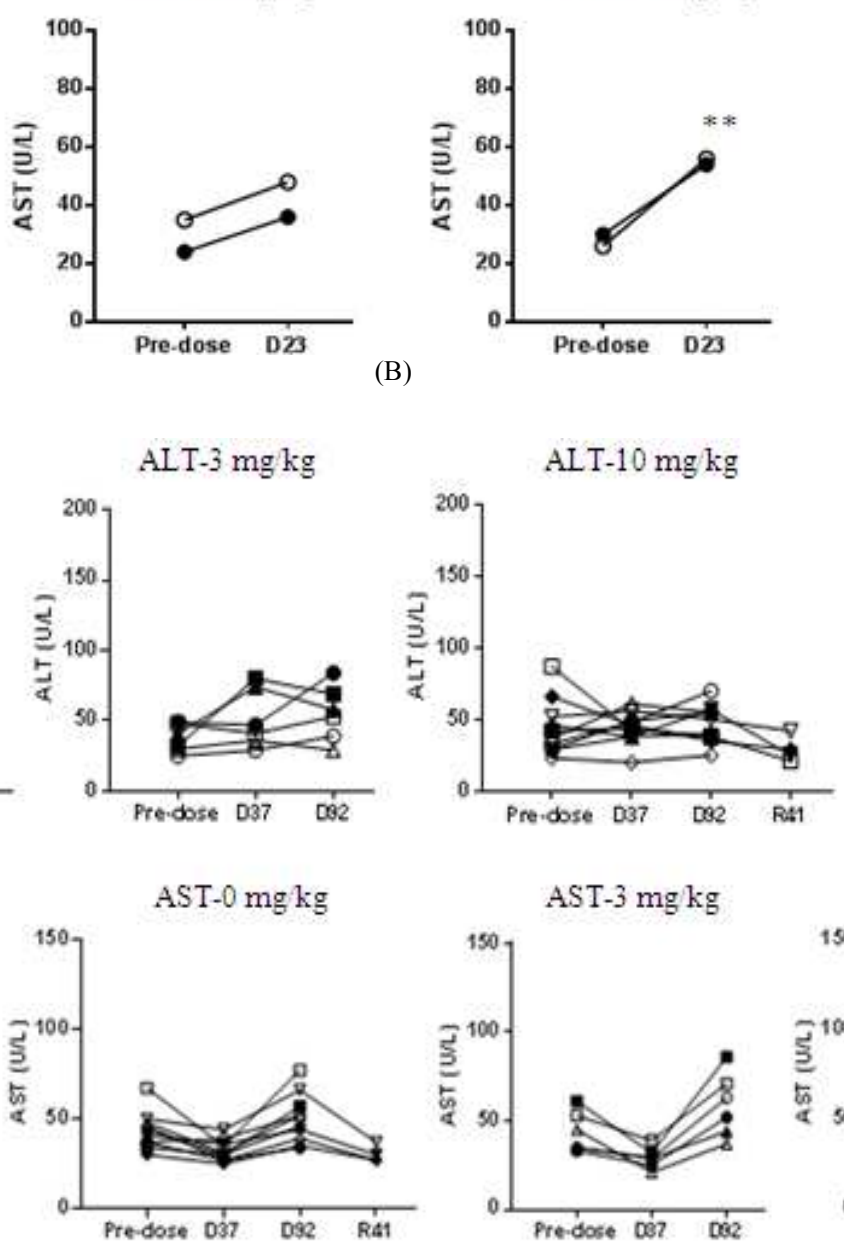

ALT-10 mg kg

AST-3 mg kg

ALT-3 mg kg

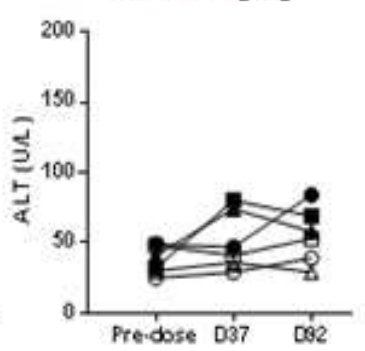

AST-0 mg kg

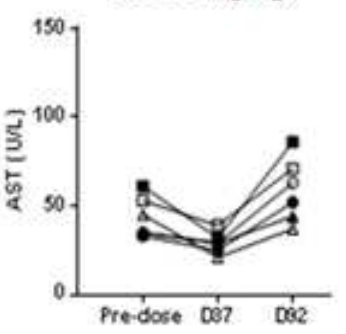

ALT-100 mg kg

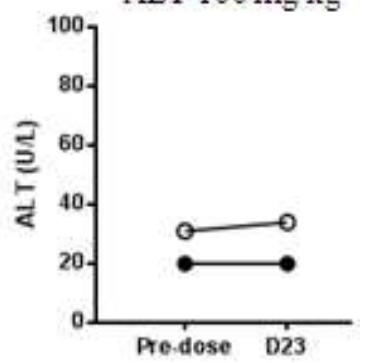

AST-100 mg kg

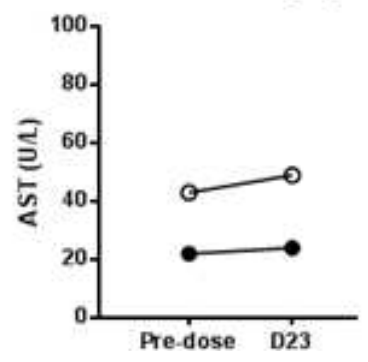

ALT-30 mg kg

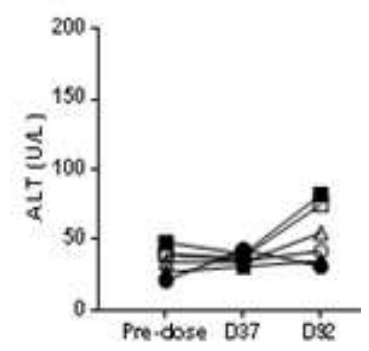

AST-10 mg kg

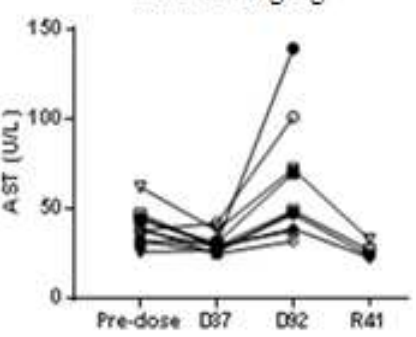



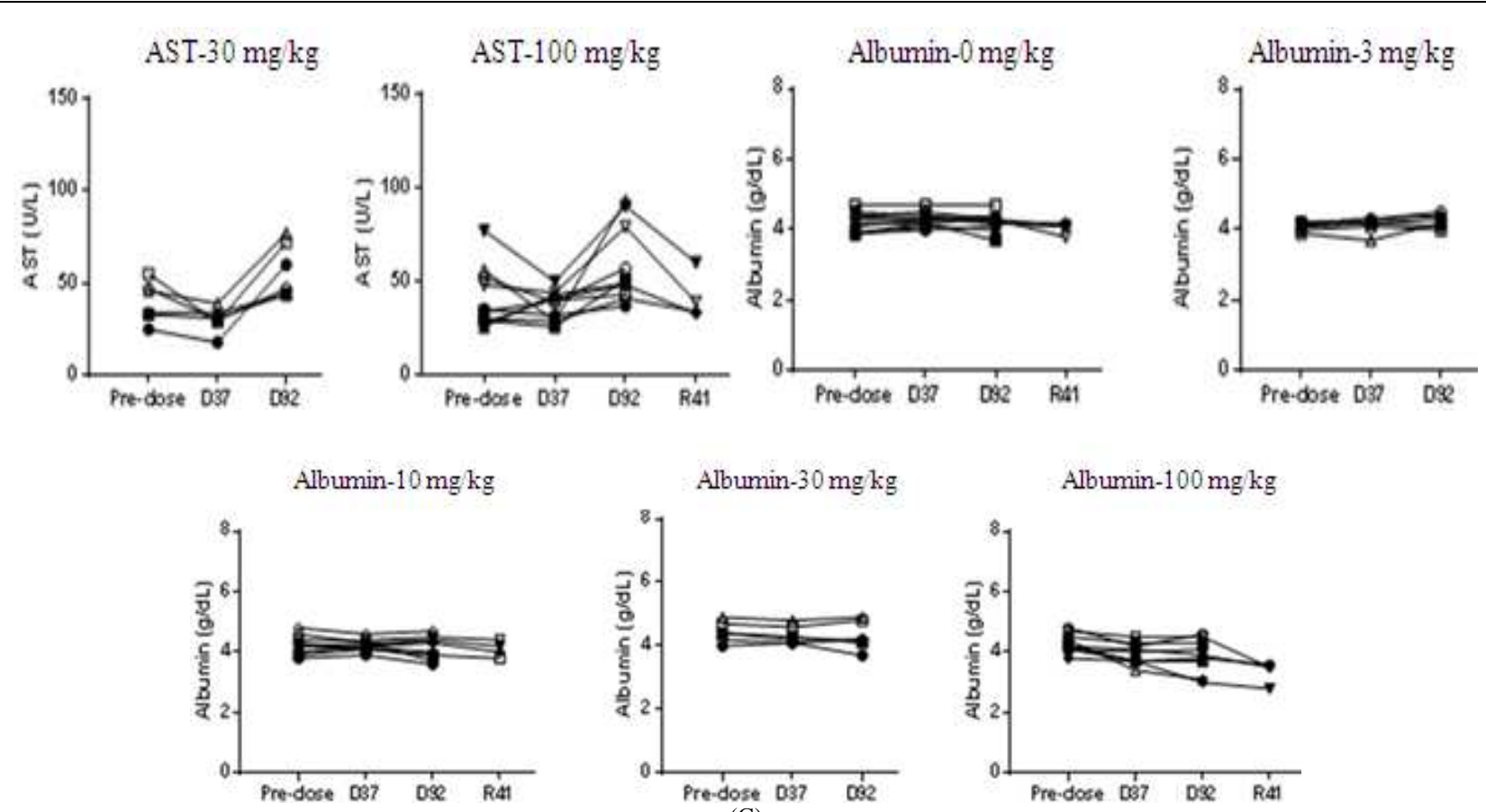

Fig. 2: Enavatuzumab elicited modest pharmacodynamic changes in cynomolgus monkeys (A) IL-6 and RANTES levels were measured in serum samples collected from cynomolgus monkeys pre - dose and at various time points after the first dose in a one-month tolerability study. The dashed line indicates the lower limit of detection for IL-6. Animal ID numbers are indicated on the X axis; animals 11, 13, 15 and 17 were female and animals 12, 14, 16 and 18 were male, B and C. Alanine Transaminase (ALT) and Aspartate Transaminase (AST) levels were measured in serum samples from cynomolgus monkeys in (B) a onemonth tolerability study and (C) a 13 - week toxicity study. Closed symbols: male animals; open symbols: female animals. Increases in ALT and AST in the $30 \mathrm{mg} / \mathrm{kg}$ group were statistically significant $\left({ }^{*} \mathrm{p}=0.01 ;{ }^{* *} \mathrm{p}=0.005\right)$

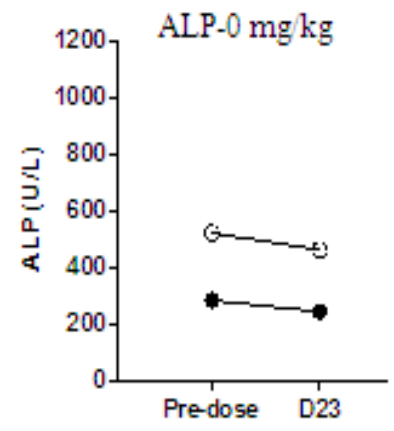

Bilinubin-0 mg $\mathrm{kg}$

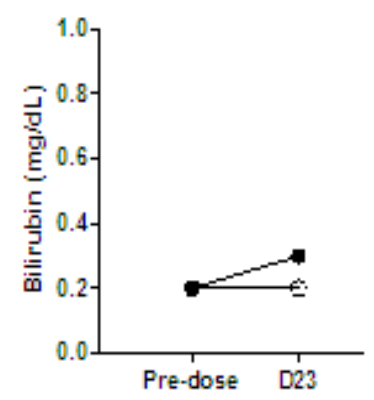

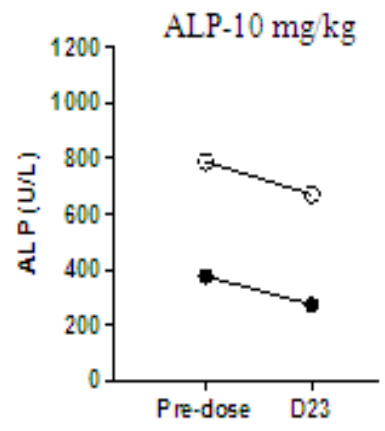

Bilirubin-10 $\mathrm{mg} / \mathrm{kg}$

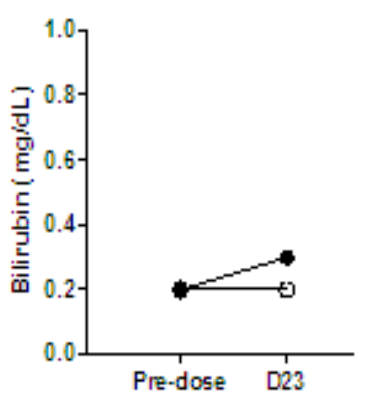

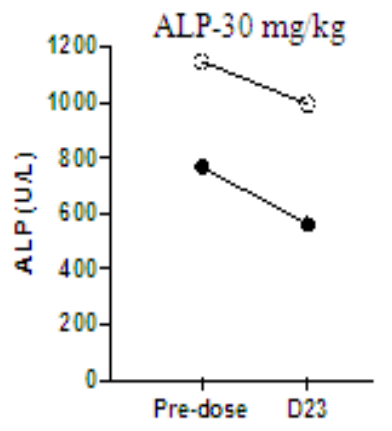

Bilirubin-30 $\mathrm{mg} / \mathrm{kg}$

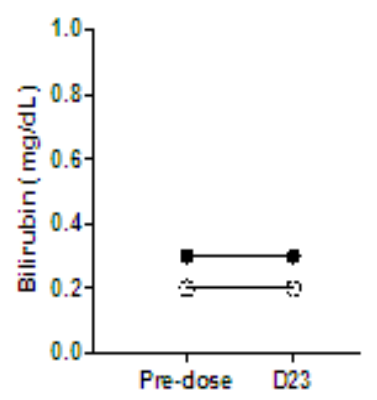

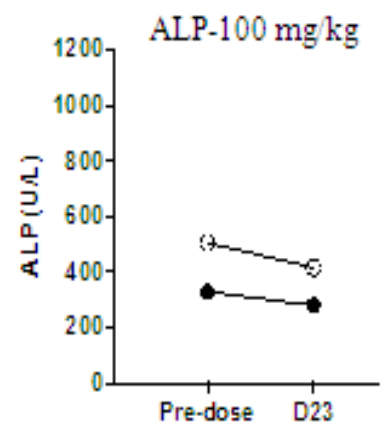

Bilirubin-100 mg kg

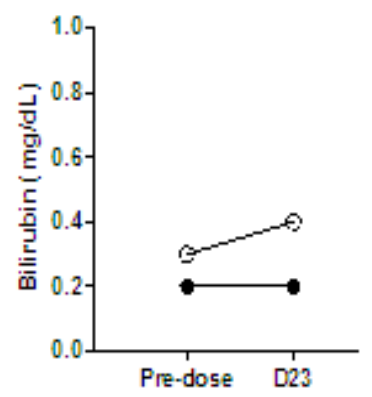



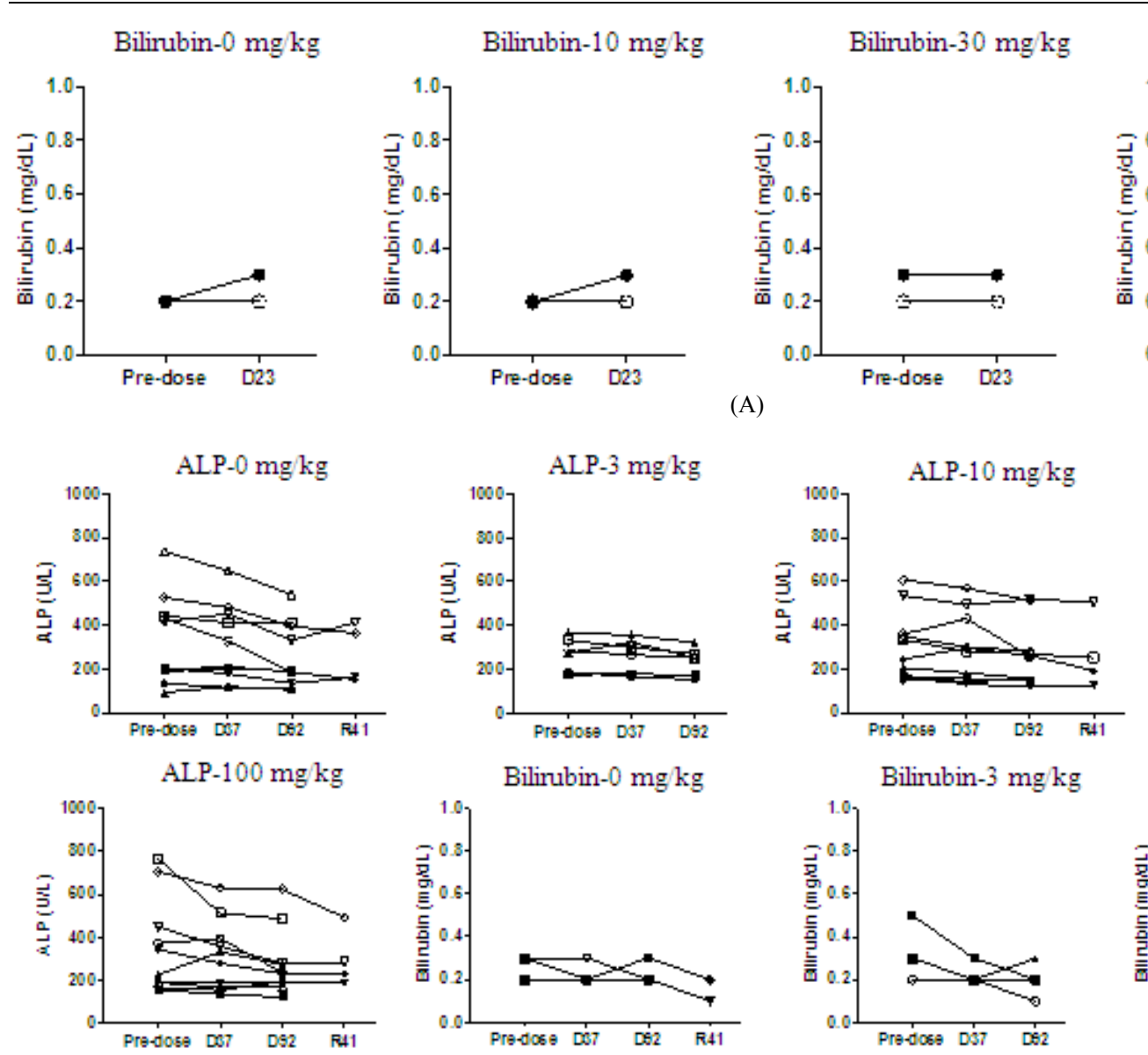

(A)
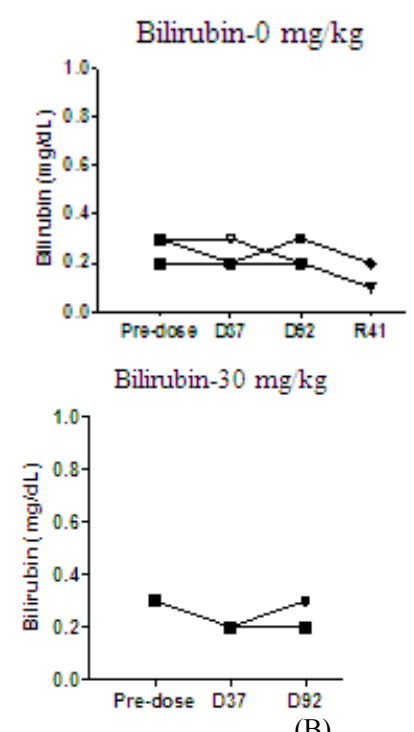

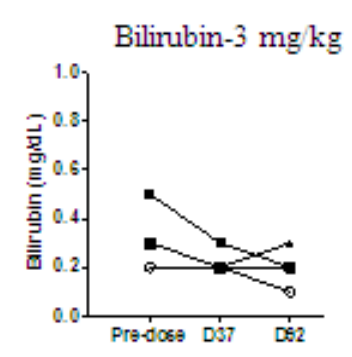

Bilirubin-100 mg kg

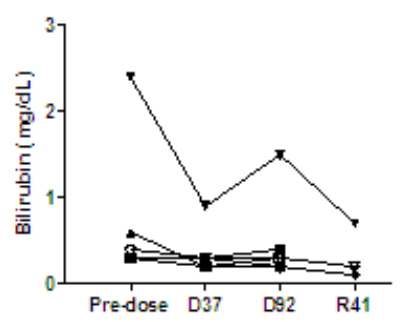

(B)
Bilinubin-100 mg kg
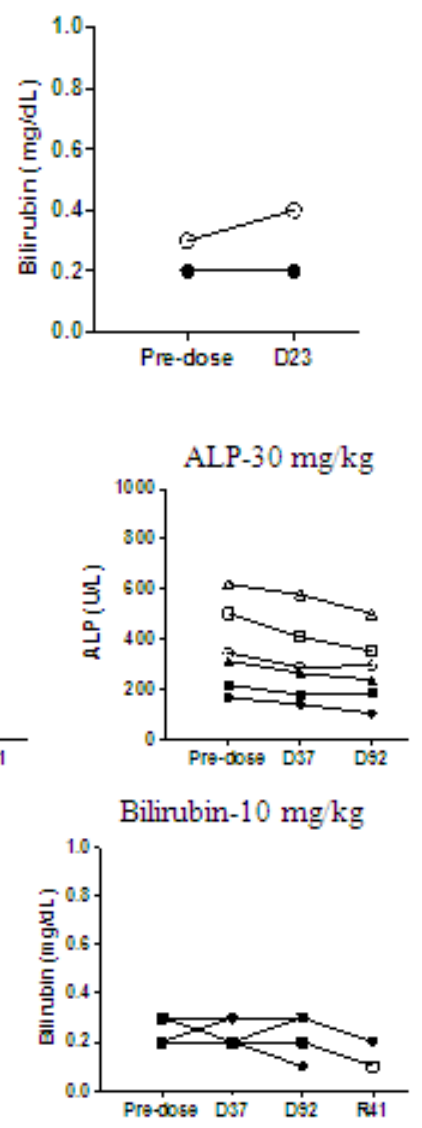

Fig. S2: Additional liver function measurements after enavatuzumab treatment of cynomolgus monkeys, A and B. Alkaline Phosphatase (ALP), bilirubin and albumin levels were measured in serum samples from cynomolgus monkeys in (A) a one-month tolerability study and (B) a 13- week toxicity study. Closed symbols: male animals; open symbols: Female animals. No significant changes in ALP, bilirubin, or albumin levels were observed in enavatuzumab - treated animals in the tolerability study and no significant changes in ALP or bilirubin levels were detected in the 13-week toxicity study

Table 1: Bile duct hyperplasia detected by histopathology in enavatuzumab-treated cynomolgus monkeys

\begin{tabular}{|c|c|c|c|c|c|c|c|c|c|c|}
\hline \multirow{3}{*}{$\begin{array}{l}\text { Dose level } \\
(\mathrm{mg} / \mathrm{kg})\end{array}$} & \multicolumn{10}{|c|}{ Necropsy day } \\
\hline & \multicolumn{5}{|c|}{ D92 } & \multicolumn{5}{|c|}{$\mathrm{R} 170$} \\
\hline & $\mathrm{N}$ & no findings & minimal & mild & moderate & $\mathrm{N}$ & no findings & minimal & mild & moderate \\
\hline 0 & 6 & 6 & 0 & 0 & 0 & 4 & 4 & 0 & 0 & 0 \\
\hline 3 & 6 & 3 & 1 & 2 & 0 & 0 & & & & \\
\hline 10 & 6 & 3 & 1 & 0 & 2 & 4 & 2 & 2 & 0 & 0 \\
\hline 30 & 6 & 0 & 4 & 1 & 1 & 0 & & & & \\
\hline 100 & 6 & 1 & 2 & 2 & 1 & 4 & 1 & 1 & 1 & 1 \\
\hline
\end{tabular}

For each dosing group and for each necropsy day (D92, 7 days after the last dose, or R170, 24 weeks after the last dose), the number of animals exhibiting the level of bile duct hyperplasia is indicated. Severity scale: No findings $<$ minimal $<$ mild $<$ moderate $<$ severe 
This finding appeared to be related to enavatuzumab treatment, as no animals in the vehicle-dosed group exhibited bile duct hyperplasia. Moreover, the finding appeared to be dose-related, as the incidence and severity increased with antibody dose level. Given that bile duct hyperplasia was observed in all dosing groups, no NOAEL (no observed adverse effect level) was identified in the study.

TweakR Expression was Increased in Liver Samples from Cancer Patients and was Associated with Tissue Inflammation

In a Phase 1 study in cancer patients, elevations in serum cytokines and liver enzymes were observed at significantly lower doses of enavatuzumab than what had been observed in cynomolgus monkeys. Thus we reasoned that liver tissues in human cancer patients may be sensitized to enavatuzumab. To explore this further, we examined liver tissues from five cancer patients and ten healthy human donors. In comparison to liver tissues from healthy donors, liver samples from the cancer patients exhibited marked infiltration of immune cells, accompanied by elevated expression of TweakR, especially notable in the bile ducts (Fig. 3).
This finding was observed in all samples from cancer patients, regardless of whether the patients had received prior chemotherapy treatment.

Enavatuzumab Induced Cytokine Release from Hepatocytes and in the Presence of Immune Cells, also Induced Release of Liver Enzymes

To explore further the impact of enavatuzumab on the liver under inflammatory conditions, we established an in vitro model to evaluate enavatuzumab-treated hepatocytes in the presence versus the absence of immune cells. Enavatuzumab treatment of cultured hepatocytes had no significant impact on the release of cytokines or chemokines within the timecourse of the assay (Fig. 4A, Supplemental Table S2). However, crosslinking enavatuzumab with secondary antibody increased release of several cytokines and chemokines, including GM-CSF and IL-6. Other cytokines, including IFN $\gamma$ or $\mathrm{TNF} \alpha$, were not impacted by enavatuzumab. Enavatuzumab treatment did not elevate ALT or AST levels released from the cultured hepatocytes (Fig. 4B).

Table S2: Crosslinking enavatuzumab significantly increased cytokine release from cultured hepatocytes

\begin{tabular}{|c|c|c|c|c|}
\hline & \multirow[b]{2}{*}{ huIgG1 } & \multirow[b]{2}{*}{ Enavatuzumab } & \multicolumn{2}{|c|}{+ xlinker } \\
\hline & & & huIgG1 & enavatuzumab \\
\hline Eotaxin & 28 & 28 & 28 & 32 \\
\hline G-CSF & BLQ & 22 & 49 & 445 \\
\hline GM-CSF & 40 & 58 & 77 & $541 *$ \\
\hline $\mathrm{IFN} \alpha 2$ & 15 & 35 & 28 & 25 \\
\hline $\mathrm{IFN} \gamma$ & 0.4 & 3 & 1 & 2 \\
\hline IL-1 $\alpha$ & 2 & 3 & 2 & 6 \\
\hline IL-1 $\beta$ & 1 & 3 & 1 & 2 \\
\hline IL-2 & BLQ & 1 & BLQ & BLQ \\
\hline IL-3 & BLQ & 0.4 & BLQ & BLQ \\
\hline IL-4 & BLQ & BLQ & BLQ & BLQ \\
\hline IL-5 & BLQ & BLQ & BLQ & BLQ \\
\hline IL-6 & 5 & 14 & 24 & $84 *$ \\
\hline IL-7 & BLQ & 1 & 1 & 2 \\
\hline IL-8 & ALQ & ALQ & ALQ & ALQ \\
\hline IL-10 & BLQ & BLQ & BLQ & BLQ \\
\hline IL-12p40 & 3 & 15 & 2 & 2 \\
\hline IL-12p70 & 1 & 5 & 1 & 2 \\
\hline IL-13 & BLQ & 0.2 & BLQ & BLQ \\
\hline IL-15 & BLQ & 1 & 0.2 & 1 \\
\hline IL-17 & 0.5 & 1 & 0.4 & 0.5 \\
\hline IP-10 & 15 & 39 & 38 & $188^{*}$ \\
\hline MCP-1 & ALQ & ALQ & ALQ & ALQ \\
\hline MIP- $1 \alpha$ & 3 & 8 & 19 & 39 \\
\hline MIP-1 $\beta$ & 5 & 9 & 25 & 50 \\
\hline TNF- $\alpha$ & BLQ & 0.4 & 1 & 5 \\
\hline TNF $\beta$ & BLQ & BLQ & BLQ & BLQ \\
\hline
\end{tabular}

Mean cytokine and chemokine concentrations (in $\mathrm{pg} / \mathrm{mL}$ ) measured in supernatants in a representative experiment *Cytokine levels were significantly increased by enavatuzumab treatment compared to isotype control $(\mathrm{p}<0.05)$. ALQ: Above the limit of quantification, BLQ: Below the limit of quantification 


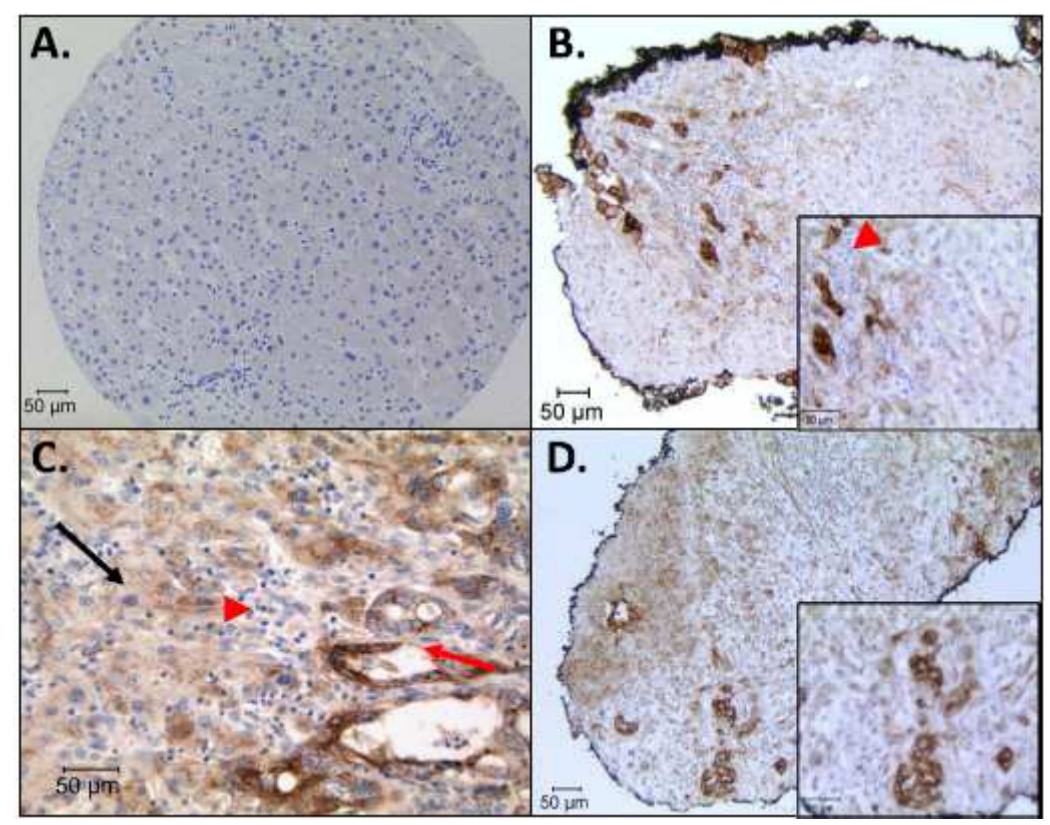

Fig. 3: Liver inflammation in cancer patients is associated with elevated TweakR expression. FFPE liver tissues from a healthy human donor (A) and 3 cancer patients (B-D) were stained for TweakR expression by immunohistochemistry. In B and C, infiltrating immune cells are indicated by red arrowheads. In C, normal hepatocytes and tumor cells are indicated by black and red arrows, respectively. In D, TweakR expression on bile duct is shown in the inset. Panels A, B and D are shown in 200X magnification, panel $\mathrm{C}$ is shown in 400X magnification and insets are shown in 600X magnification

GM-CSF

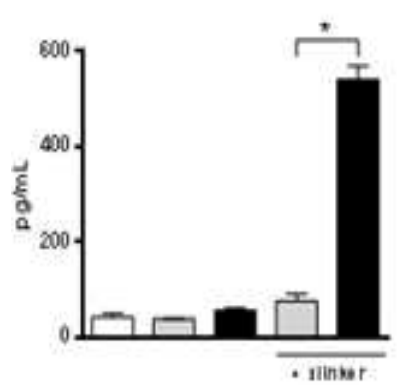

IL-6

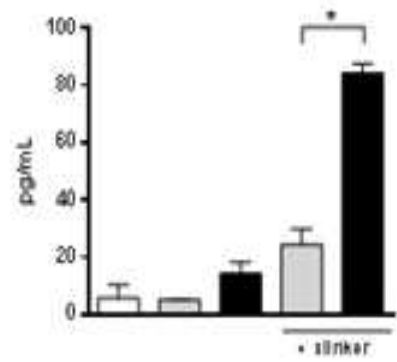

IFNy

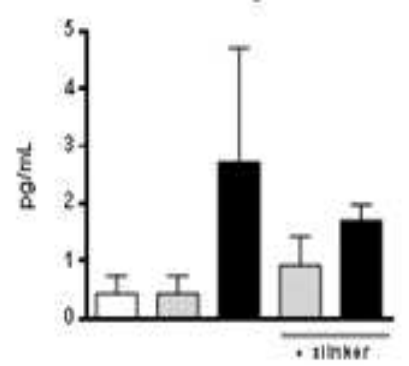

TNF $\alpha$

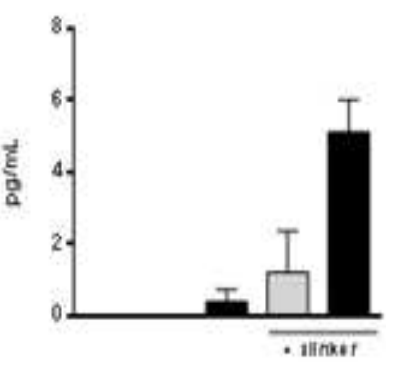

ALT

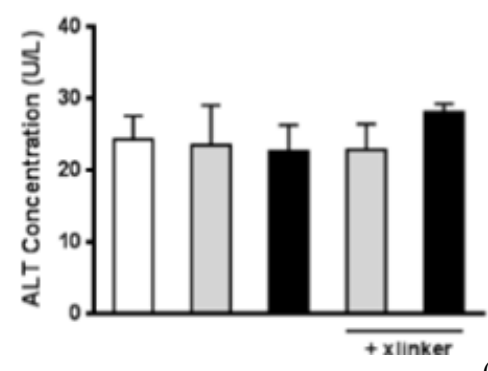

AST

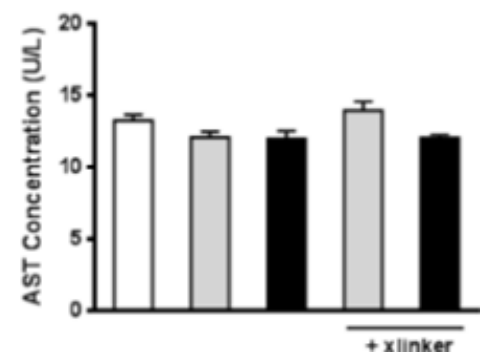

(B)

Fig. 4: Enavatuzumab stimulated cytokine and chemokine release from hepatocytes but did not induce release of liver enzymes. Hepatocytes were untreated (white bars) or were incubated with enavatuzumab (black bars) or an isotype control antibody (grey bars) +/- an anti-human Fc crosslinking antibody (+ xlinker) for $24 \mathrm{~h}$, after which supernatants were collected and assessed for (A) cytokines and (B) Alanine Transaminase (ALT) and Aspartate Transaminase (AST) levels. *Enavatuzumab treatment significantly increased cytokine levels $(\mathrm{p}<0.05)$ 
In co-cultures of immune cells and hepatocytes, enavatuzumab treatment significantly elevated the levels of a number of cytokines released from the cultures, including GM-CSF, IFN $\gamma$ and TNF $\alpha$ (Fig. 5A, Supplemental Table S3). Across multiple experiments using several hepatocyte and immune cell donors, enavatuzumab elicited cytokine release in all co-culture experiments, although the magnitude varied. With a subset of donors, generally in those experiments where higher levels of cytokine release were observed, enavatuzumab treatment of the co-cultures resulted in elevated ALT and AST levels in the supernatants (Fig. 5B).

Table S3: Enavatuzumab induced cytokine release in co-cultures of hepatocytes and immune cells

\begin{tabular}{|c|c|c|c|c|}
\hline & \multirow[b]{2}{*}{ huIgG1 } & \multirow[b]{2}{*}{ enavatuzumab } & \multicolumn{2}{|c|}{ + Dexamethasone } \\
\hline & & & huIgG1 & enavatuzumab \\
\hline Eotaxin & 41 & 67 & 28 & $51 * \dagger$ \\
\hline G-CSF & 1841 & 2557 & 1040 & 1704 \\
\hline GM-CSF & 45 & $194 *$ & 24 & $64 \dagger$ \\
\hline IFN $\alpha 2$ & 11 & 11 & 3 & 12 \\
\hline $\operatorname{IFN} \gamma$ & 4 & $64 *$ & 0.1 & $10 * \dagger$ \\
\hline IL- $1 \alpha$ & 129 & $348 *$ & 105 & $262 * \dagger$ \\
\hline IL-1 $\beta$ & 45 & 91 & 4 & 9 \\
\hline IL-2 & 2 & 2 & 0.1 & 0.2 \\
\hline IL-3 & BLQ & BLQ & BLQ & BLQ \\
\hline IL-4 & BLQ & BLQ & BLQ & BLQ \\
\hline IL-5 & BLQ & BLQ & BLQ & BLQ \\
\hline IL-6 & 1185 & 1980 & 105 & $396 \dagger$ \\
\hline IL-7 & 6 & 11 & BLQ & 4 \\
\hline IL-8 & ALQ & ALQ & ALQ & ALQ \\
\hline IL-10 & BLQ & $3 *$ & 12 & 12 \\
\hline IL-12p40 & 6 & $29 *$ & 1 & 10 \\
\hline IL-12p70 & 2 & $30 *$ & 0.3 & $12 * \dagger$ \\
\hline IL-13 & BLQ & 7 & BLQ & BLQ \\
\hline IL-15 & BLQ & BLQ & BLQ & BLQ \\
\hline IL-17 & 1 & 1 & 0.2 & 0.5 \\
\hline IP-10 & 5053 & ALQ & 4828 & ALQ \\
\hline MCP-1 & 5639 & ALQ & 732 & ALQ \\
\hline MIP-1 $\alpha$ & 9 & $197 *$ & BLQ & $34 \dagger$ \\
\hline MIP-1 $\beta$ & 75 & $3242 *$ & 47 & $1909 * \dagger$ \\
\hline TNF- $\alpha$ & 11 & $144 *$ & 3 & $63 * \dagger$ \\
\hline TNF $\beta$ & BLQ & BLQ & BLQ & BLQ \\
\hline
\end{tabular}

Mean cytokine and chemokine concentrations (in $\mathrm{pg} / \mathrm{mL}$ ) measured in supernatants in a representative experiment *Cytokine levels were significantly increased by enavatuzumab treatment compared to isotype control $(p<0.05) \dagger$ Cytokine levels in enavatuzumabtreated samples were significantly reduced by dexamethasone pre-treatment $(p<0.05)$, BLQ: Below the limit of quantification
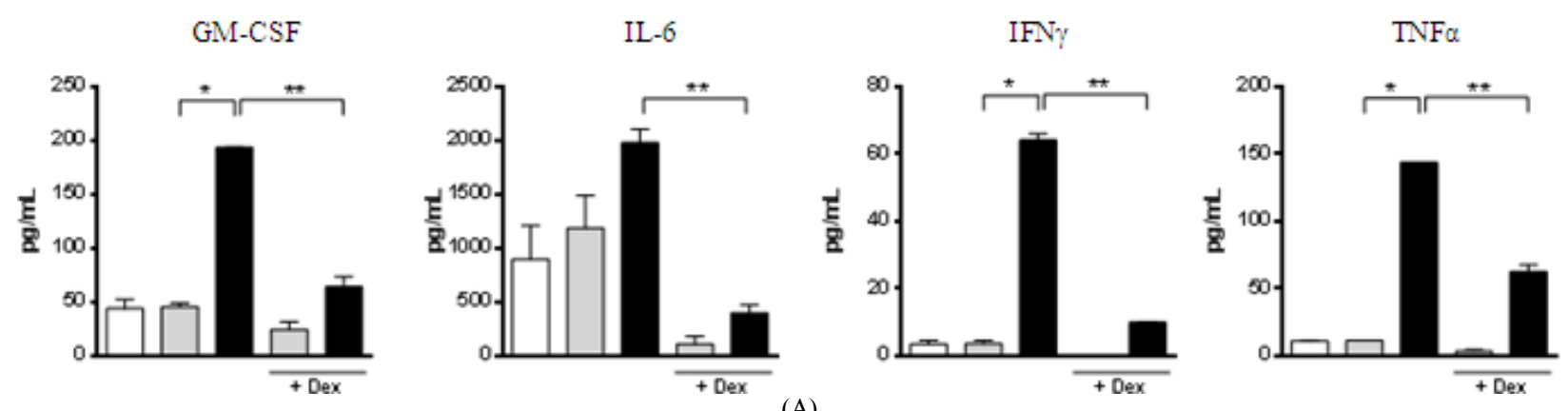

(A) 

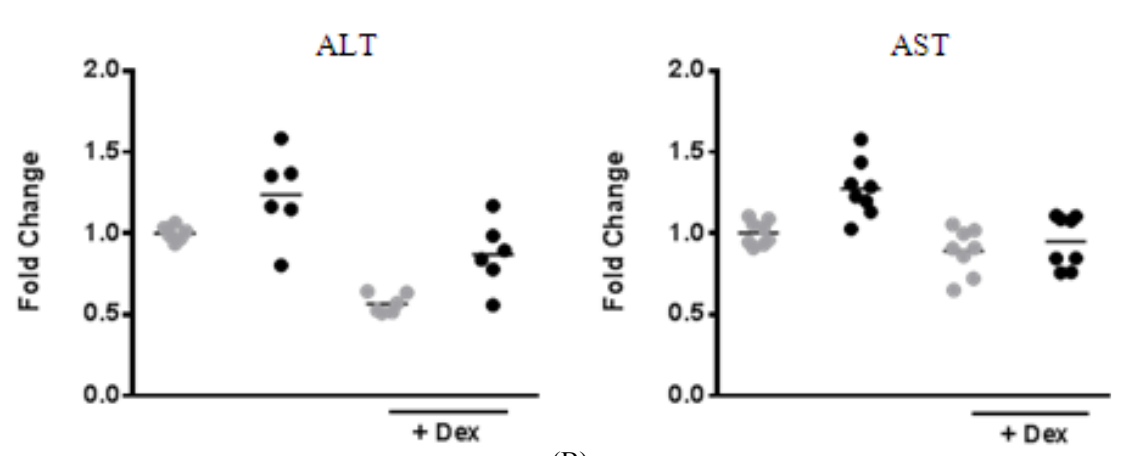

(B)

GM-CSF
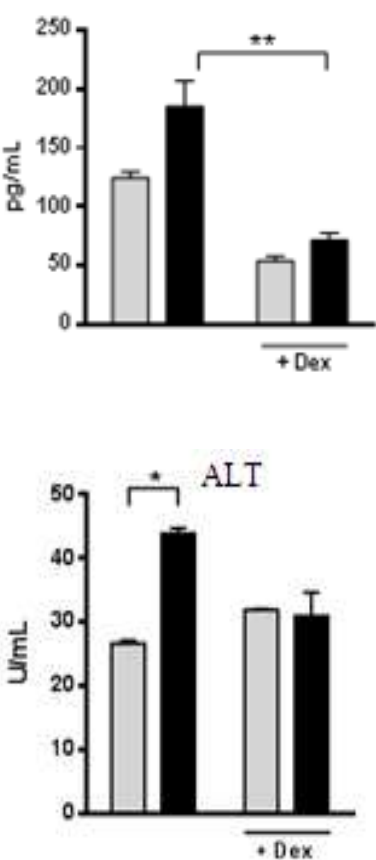

IL-6

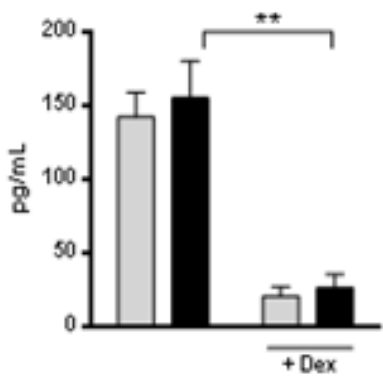

(C)

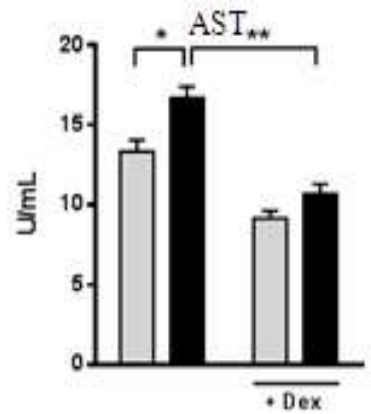

(D)

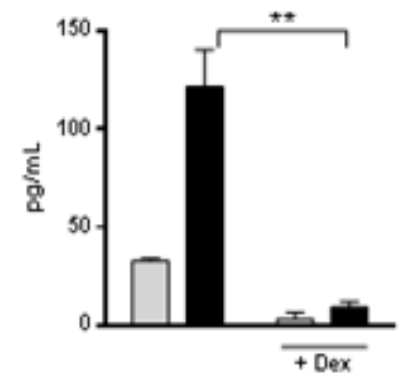

$4 \mathrm{~h}$

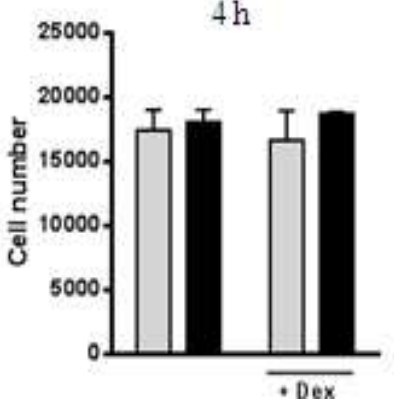

(E)
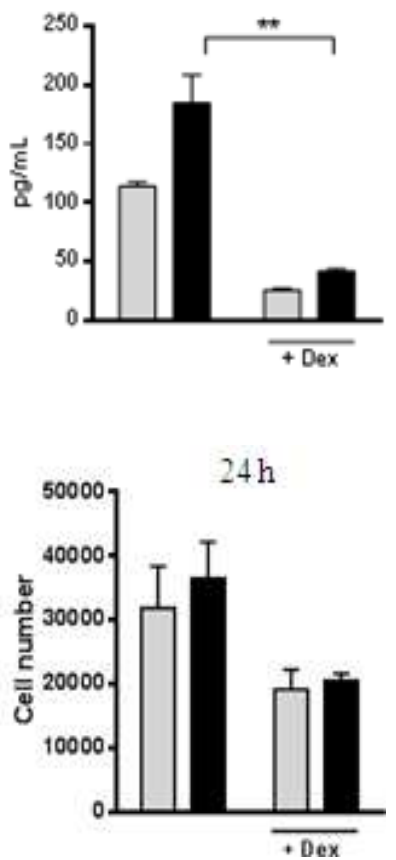

Fig. 5: Enavatuzumab stimulated release of cytokines and liver enzymes in hepatocytes cultured with immune cells (A) Hepatocytes and PBMCs were co-cultured for $24 \mathrm{~h}$ in the presence of enavatuzumab (black bars), an isotype control antibody (grey bars), or no antibody (white bars), after which cytokine levels were quantified in the supernatants. Samples treated with dexamethasone prior to antibody treatment are indicated, (B) Supernatants from hepatocyte: PBMC co-cultures were analyzed for ALT and AST levels $24 \mathrm{~h}$ after treatment with enavatuzumab (black circles) or an isotype control antibody (grey circles). Each circle represents a different hepatocyte and/or PBMC donor. The data were normalized to the mean of the isotype treated group (without dexamethasone) and fold change is indicated (C-E) Hepatocytes in the lower chamber and PBMCs in the upper chamber of a Transwell ${ }^{\circledR}$ assay were incubated with enavatuzumab (black bars) or isotype control antibody (grey bars) for $4 \mathrm{~h}$ or $24 \mathrm{~h}$, after which (C) the levels of cytokines were measured in the supernatants; (D) ALT and AST levels were measured; or (E) the number of immune cells that had migrated to the lower chamber was quantified. For dexamethasone - treated samples, hepatocytes and PBMCs were treated separately with $10 \mu \mathrm{M}$ dexamethasone prior to placement in the Transwell ${ }^{\circledR}$ and addition of antibody. * Enavatuzumab treatment significantly increased cytokine or liver enzyme levels $(\mathrm{p}<0.05)$. ** Dexamethasone treatment significantly decreased cytokine or liver enzyme levels $(p<0.05)$

Enavatuzumab treatment of cultured immune cells alone did not elicit cytokine release (Supplemental Table S4), suggesting that the impact of enavatuzumab on the hepatocyte: PBMC co-cultures was a result of enavatuzumab binding and activating TweakR expressed on the hepatocytes. However, cytokines and/or chemokines released by the hepatocytes in response to enavatuzumab treatment likely activated the immune cells to elicit 
secondary effects, resulting in the elevated proinflammatory cytokines and ALT/AST release. To explore further the potential relationship between cytokines and/or chemokines and liver enzyme release, hepatocyte: PBMC co-cultures were pre-treated with dexamethasone to suppress cytokine release. Enavatuzumab treatment of dexamethasone-treated samples resulted in significantly reduced levels of cytokinesand AST levels (Fig. 5A-B, Supplemental Table S3).

Enavatuzumab was also evaluated on hepatocyte and PBMC cultures in a Transwell ${ }^{\circledR}$ assay, where the immune cells in the upper chamber were able to migrate towards enavatuzumab-treated hepatocytes in the lower chamber. In this assay, enavatuzumab treatment resulted in a trend toward elevated cytokine levels but did not reach significance (Fig. 5C, Supplemental Table S5). As observed in the coculture assay, enavatuzumab stimulated ALT and AST release in a subset of donors in the Transwell ${ }^{\circledR}$ assay (Fig. 5D) and dexamethasone pre-treatment of the cells significantly suppressed cytokine and ALT/AST elevations. Quantification of the migrating immune cells revealed that enavatuzumab treatment had no impact on the migration of immune cells toward hepatocytes (Fig. 5E). However, dexamethasone pretreatment resulted in a significant reduction in the number of the baseline immune cells that had migrated after $24 \mathrm{~h}$.

\section{Dexamethasone did not Impact the Anti-Tumor Activity of Enavatuzumab}

The finding that dexamethasone effectively reduced enavatuzumab-stimulated cytokine/chemokine and ALT/AST levels in vitro suggested that steroid pre-treatment may be a means to alleviate enavatuzumab-induced liver toxicity in vivo. Thus, we evaluated the impact of dexamethasone on the anti-tumor activity of enavatuzumab in mouse xenograft models. As previously described, enavatuzumab elicits potent antitumor activity through two mechanisms of action: direct inhibition of tumor cell growth and antibody-dependent cellular cytotoxicity (ADCC) (Culp et al., 2010). We evaluated the impact of dexamethasone on the anti-tumor activity of enavatuzumab in two xenograft models: H358, an ADCC-independent model and SN12C, an ADCCdependent model (Culp et al., 2010; Purcell et al., 2014). In both models, dexamethasone modestly inhibited tumor growth and either had no impact on enavatuzumab activity or modestly enhanced the anti-tumor activity of enavatuzumab (Supplemental Fig. S3).

Table S4: Enavatuzumab did not induce cytokine release from PBMCs

\begin{tabular}{llr}
\hline & huIgG1 & enavatuzumab \\
\hline Eotaxin & 26 & 26.0 \\
GM-CSF & 8 & 8.0 \\
IFN $\gamma$ & 13 & 10.0 \\
IL-1 $\alpha$ & 31 & 35.0 \\
IL-1 $\beta$ & 17 & 10.0 \\
IL-2 & 6 & 4.0 \\
IL-3 & 5 & 5.0 \\
IL-4 & 6 & 5.0 \\
IL-5 & 4 & 3.5 \\
IL-6 & 92 & 123.0 \\
IL-7 & 4 & 4.0 \\
IL-8 & 2398 & 3306.0 \\
IL-10 & 35 & 32.0 \\
IL-12p40 & 178 & 171.0 \\
IL-12p70 & 6 & 5.0 \\
IL-13 & 2 & 2.0 \\
IL-15 & 7 & 6.0 \\
IP-10 & 58 & 130.0 \\
MCP-1 & 52 & 69.0 \\
MIP-1 $\alpha$ & 137 & 197.0 \\
RANTES & 12250 & 12880.0 \\
TNF $\alpha$ & 6 & 5.0 \\
\hline M-an & 170 &
\end{tabular}

Mean cytokine and chemokine concentrations (in $\mathrm{pg} / \mathrm{mL}$ ) measured in supernatants in a representative experiment 
$\underline{\text { Table S5: Enavatuzumab induced cytokine release from Transwell }{ }^{\circledR} \text { assays containing hepatocytes and PBMCs }}$

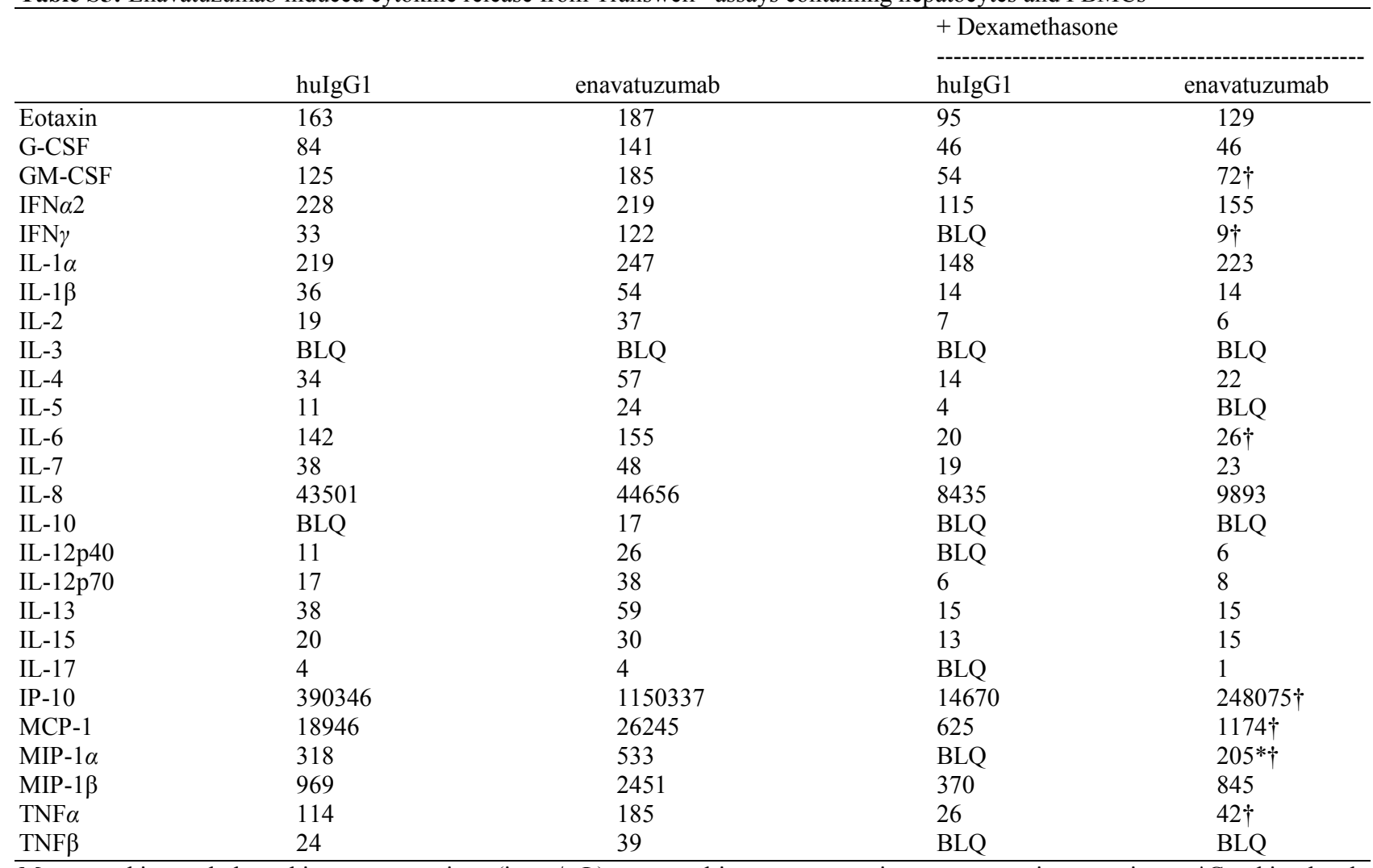

Mean cytokine and chemokine concentrations (in pg/mL) measured in supernatants in a representative experiment *Cytokine levels were significantly increased by enavatuzumab treatment compared to isotype control $(p<0.05) \dagger$ Cytokine levels in enavatuzumabtreated samples were significantly reduced by dexamethasone pre-treatment $(\mathrm{p}<0.05)$, BLQ: Below the limit of quantification

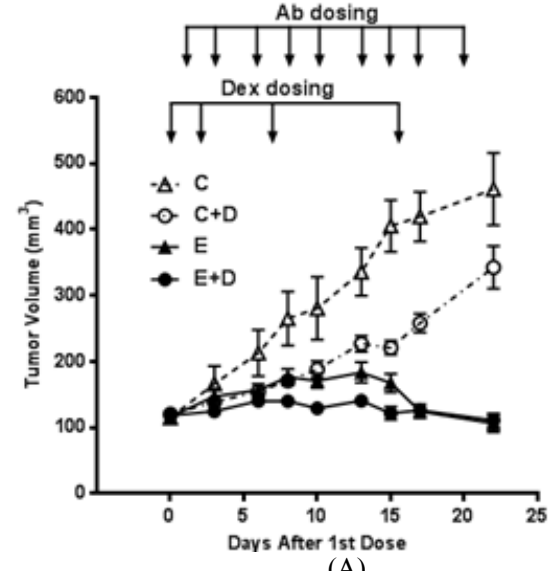

(A)

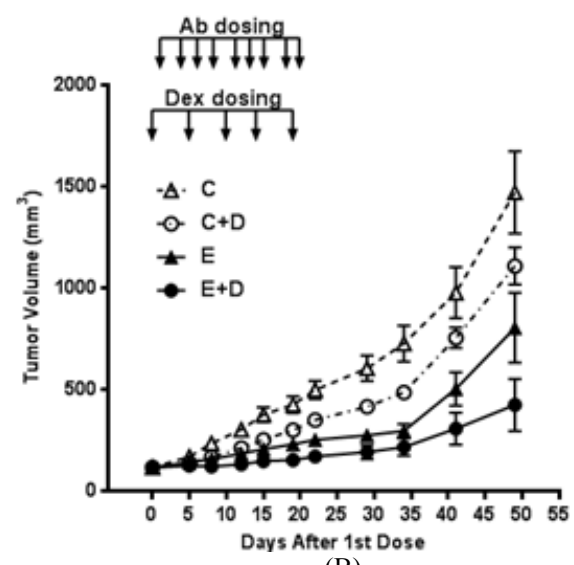

(B)

Fig. S3: Dexamethasone did not impact the anti-tumor activity of enavatuzumab, ICR-SCID mice bearing (A) NCI-H358 or (B) SN12C xenograft tumors were treated with Enavatuzumab (E) or an isotype control antibody (C) $(1 \mathrm{mg} / \mathrm{kg})+/-$ dexamethasone (D) $(5 \mathrm{mg} / \mathrm{kg})$. Dosing days are indicated on each graph and tumor volumes on each day of measurement are indicated as the mean $+/-$ SEM

\section{Discussion}

This report describes the preclinical studies evaluating the mechanism of liver toxicity induced by enavatuzumab. No liver toxicity was observed in the one-month tolerability study in cynomolgus monkeys and in the 13-week toxicity study, enavatuzumab induced ALT elevations only at the highest dose level 
tested, $100 \mathrm{mg} / \mathrm{kg}$. However, in this latter study, bile duct hyperplasia was observed at all dose levels. This histopathology finding is consistent with a previous report describing the impact of activating the TWEAK/TweakR pathway on the liver in mice. TWEAK transgenic mice exhibit hyperplasia of oval cells, which are liver progenitor cells, but the animals do not exhibit liver enzyme elevations (Jakubowski et al., 2005). In the same study, the authors demonstrated that ectopic expression of TWEAK in the liver of adult mice resulted in oval cell and biliary duct hyperplasia without significant liver damage. While activating the TweakR pathway in cynomolgus and mouse exhibited similar signs of liver toxicity by histopathology, these preclinical studies greatly underestimated the sensitivity of human cancer patients to liver toxicity induced by enavatuzumab, as measured by ALT and AST elevations. Whether enavatuzumab induced hyperplasia in the livers of the patients in the Phase 1 study is unknown, as post-dose liver biopsies were not available.

One possible explanation for the disparity in sensitivities of human and cynomolgus to enavatuzumab is innate differences in the sensitivities of the two species to the antibody. Using several cynomolgus tissue sources we found the amino acid sequences between human and cynomolgus to be identical. This is in contrast to a previous report describing cynomolgus TweakR as $98 \%$ identical to human TweakR (Zheng and Burkly, 2008). However, enavatuzumab bound TweakR in both species similarly and induced cytokine release in the two species. Another and perhaps more likely, explanation for the increased sensitivity of humans to enavatuzumab is that the architecture of the liver in cancer patients is altered, as observed by infiltration of immune cells and elevated TweakR expression, either of which may contribute to enavatuzumab sensitivity.

In the liver tissues of cancer patients, the areas of inflammation in the liver were accompanied by elevated TweakR expression. We observed a similar increase in TweakR expression in a liver sample from a patient with liver inflammation that exhibited bile duct dilatation and mild fibrosis but not from a patient who exhibited noninflammatory angiomyolipoma (data not shown). The elevation of TweakR expression in inflammatory tissues has also been described in multiple disease states, including lupus, rheumatoid arthritis, psoriatic arthritis and multiple sclerosis (Lu et al., 2011; van Kuijk et al., 2010; Dharmapatni et al., 2011; Serafini et al., 2008).

In experiments designed to explore the mechanism of enavatuzumab-induced liver toxicity, co-cultures of hepatocytes and immune cells were used to model the impact of enavatuzumab on cytokine and ALT/AST release. A range of methods for evaluating liver toxicity have previously been described, including use of liver tissue slices and 2D or 3D co-cultures of hepatocytes with other cell types (Soldatow et al., 2013; Lake and Price, 2013; Sahi et al., 2010; Gomez-Lechon et al., 2010). In such models, where hepatocytes are cocultured with other cell types, the relevant cell-cell interactions that occur in vivo are replicated in vitro. In the enavatuzumab Phase 1 study, liver toxicity was largely limited to ALT/AST elevations, suggesting that the toxicity elicited by enavatuzumab was likely due to direct stimulation of hepatocytes, consistent with the expression of TweakR on hepatocytes in cancer patients. Given the likely direct effect of enavatuzumab on hepatocytes and the inflammation observed in liver samples from cancer patients, a co-culture of hepatocytes and PBMCs was able to replicate some of the relevant cell-cell interactions in the livers of cancer patients. However, a more complex model, such as the use of tissue slices from cancer patients, would contain additional cell types and the effect of enavatuzumab in such a system would be interesting to explore.

Interestingly, cultured normal hepatocytes expressed TweakR, unlike hepatocytes that reside in non-diseased livers. The elevated expression of TweakR on cultured hepatocytes may be a result of the culture conditions, as growth factors in culture media have been shown to upregulate TweakR expression (Wiley et al., 2001). Fortuitously, TweakR expression on hepatocytes cultured in the absence of immune cells provided the opportunity to evaluate the direct effect of enavatuzumab treatment of hepatocytes.

Immune cells provide multiple potential mechanisms for impacting the functional activity of enavatuzumab on hepatocytes, one of which is to crosslink the antibody through Fc receptors expressed on the immune cells. Mimicking that function with a secondary antibody dramatically increased the level of cytokines released by enavatuzumab; this finding is consistent with increased functional activity of enavatuzumab and other TNFRSF agonist antibodies when crosslinked with a secondary antibody or Protein A (Chao et al., 2013; Salzmann et al., 2013; Chuntharapai et al., 2001; White et al., 2013; Li et al., 2006; Dhein et al., 1992; Chodorge et al., 2012). However, crosslinking the antibody did not increase ALT/AST release, which was only observed when immune cells were present. In addition, in the presence of immune cells, the type of cytokines induced by enavatuzumab was altered. Crosslinked enavatuzumab resulted largely in the release of the chemokines GM-CSF, IL-6 and IP-10; however in co-cultures of hepatocytes and immune cells, additional chemokines and the pro-inflammatory cytokines, IFN $\gamma$ and TNF $\alpha$, were strikingly enhanced by enavatuzumab treatment. Such a qualitative change may be due, at least in part, to an allogeneic response, as the hepatocytes and PBMCs were not from matched donors. The mechanism of liver toxicity induced by 
enavatuzumab in co-cultures of hepatocytes and immune cells may be a result of ADCC or may be indirect, possibly through a cytokine-mediated effect. Our finding that dexamethasone inhibited enavatuzumab-dependent ALT/AST increases suggests that the hepatocellular toxicity was mediated at least in part by cytokines. These results indicate that immune cells likely mediate multiple functions to induce liver toxicity with enavatuzumab.

In the in vitro assays, while experiments across all PBMC and hepatocyte donors exhibited some elevations in secreted cytokines upon enavatuzumab treatment, stimulation of ALT and AST release was observed only in a subset of donors and generally in experiments where higher levels of cytokines were released. This varied response to enavatuzumab across human donors reflected the variation of liver toxicity observed in cancer patients in the Phase 1 study. Of the three patients from the Phase 1 study whose liver biopsies were tested for TweakR expression by IHC, all showed increased TweakR expression and inflammation, but only one patient had experienced liver enzyme elevations after enavatuzumab treatment (Lam et al., 2017). Thus, increased TweakR expression and/or inflammation may contribute to sensitivity to enavatuzumab, but additional factors are also likely involved in inducing liver toxicity. In the Phase 1 study, a number of cytokines were elevated in the serum of a subset of patients after enavatuzumab treatment; however, cytokine and ALT/AST elevations were not correlated (Lam et al., 2017). As multiple normal tissues express TweakR, the post-dose cytokine elevations may have arisen from the liver or other TweakR-positive tissues.

Liver toxicity is less commonly observed with biologics agents than with small molecule chemotherapy agents (Baldo, 2013; Saez et al., 2012). A notable exception is the TNF Receptor Super Family (TNFRSF), where agonist antibodies to multiple TNFRSF members have been found to induce liver toxicity with a range of incidence and severity. Agonist antibodies to CD95 (Fas) rapidly produce profound and fatal liver failure in mice (Ogasawara et al., 1993) and agonist antibodies to CD137 (4-1BB), CD40 and the TRAIL receptors DR4 and DR5, have all exhibited liver toxicity in humans, as defined by ALT and/or AST elevations in a subset of patients treated with the antibodies (Vonderheide et al., 2007; Advani et al., 2009; Tolcher et al., 2007; Plummer et al., 2007; Ascierto, 2010; Camidge et al., 2010). In the case of the CD137 antibody, which also produced Grade 4 hepatitis in some patients, the adverse events were not predicted in preclinical studies in cynomolgus monkey, but CD137 antibodies did induce liver toxicity in mice (Ascierto et al., 2010; Niu et al., 2007). The mechanisms by which the different agonists induce liver toxicity likely depend on the cell types on which the receptors and/or ligands are expressed. For several of the TNFRSF members, receptor expression has been reported on hepatocytes within either healthy liver and/or in liver disease states (Afford et al., 1999; Leifeld et al., 1999; Volkmann et al., 2007; Galle et al., 1995), while for other TNFRSF members, other cells within the liver have been shown to express the receptors (Niu et al., 2007; Leifeld et al., 1999; Schwabe et al., 2001).

Of note, only agonist antibodies targeting TNFRSF members induce liver toxicity; antagonist or blocking antibodies have not exhibited such an effect (Croft $e t$ al., 2013). Thus, the activation of downstream signaling pathways and not simply receptor binding, is key to inducing liver toxicity. TweakR signals through a functional interaction between its cytoplasmic domain and one or more intracellular TRAF proteins, a signaling mechanism common to other TNFRSF members, including CD137 and CD40. One signaling pathway downstream of the TRAFs and activated by the majority of TNFRSF members is NFKB; up regulation of this pathway often results in increased production of pro inflammatory cytokines (Younes and Kadin, 2003; Napetschnig and $\mathrm{Wu}, 2013)$. That liver toxicity has been observed with multiple TNFRSF members in cancer patients, together with our finding that the liver tissues of cancer patients often harbor inflammation, suggests that cytokines may be involved in inducing liver toxicity across this family of molecules, as has been implicated for CD137 and CD40 (Niu et al., 2007; Kimura et al., 2006). In a disease state, such as cancer, where the liver has experienced significant insult and inflammation, changes in the expression of the receptors and/or ligands likely increases the liver's sensitivity to cytokines. Given that a common downstream effect of TNFRSF stimulation is cytokine release, therapeutic agonists to this family of receptors have the potential for inducing liver toxicity in some settings; employing preclinical methods to evaluate the impact of the agent on the liver can be useful for determining the potential for inducing liver toxicity.

\section{Conclusion}

The presence of immune cells in liver tissue can provide an additional mechanism by which a drug induces liver toxicity in cancer patients, which may not be predicted from preclinical toxicity studies in healthy animals. Including studies to evaluate the impact of an agent on hepatocytes in the presence of immune cells may provide a more comprehensive analysis of the potential for liver toxicity in cancer patients. 


\section{Funding Information}

The financial support for this research was provided by AbbVie.

\section{Acknowledgment}

The design, study conduct and financial support for this research were provided by AbbVie. AbbVie participated in the interpretation of data, review and approval of the manuscript publication.

\section{Author's Contributions}

Donghee Choi: Design and execution of in vitro experiments, analysis and interpretation of results, drafting of manuscript, revision and approval of final manuscript.

Yanhong Zhu: Design of cynomolgus monkey toxicity studies and interpretation of results.

Debra T. Chao: Design of IHC analysis, interpretation of data, drafting manuscript, revision and approval of final manuscript

Mien Sho: Analysis of TwerkR expression by IHC and interpretation of results

Susan Rhodes: Analysis and determination of cynomolgus monkey TweakR DNA and protein sequence

Melvin Fox: Execution of mouse xenograft studies.

Gary C. Starling: Design of experiments, revision and approval of final manuscript

Patricia A. Culp: Conception and design of experiments, analysis and interpretation of data, drafting initial version of the manuscript, revision and approval of the final version of the manuscript.

\section{Conflict of Interest}

D Choi, DT Chao, M Sho, S Rhodes and M Fox are employees of AbbVie. Y Zhu, GC Starling and PA Culp were employees of AbbVie at the time of the study. The design, study conduct and financial support for this research were provided by AbbVie.

\section{References}

Advani, R., A. Forero-Torres, R.R. Furman, J.D. Rosenblatt and A. Younes et al., 2009. Phase I study of the humanized anti-CD40 monoclonal antibody dacetuzumab in refractory or recurrent non-Hodgkin's lymphoma. J. Clin. Oncol., 27: 4371-4377.

Afford, S.C., S. Randhawa, A.G. Eliopoulos, S.G. Hubscher and L.S. Young et al., 1999. CD40 activation induces apoptosis in cultured human hepatocytes via induction of cell surface fas ligand expression and amplifies fas-mediated hepatocyte death during allograft rejection. J. Exp. Med., 189: 441-446.
Ascierto, P.A., E. Simeone, M. Sznol, Y.X. Fu and I. Melero, 2010. Clinical experiences with antiCD137 and anti-PD1 therapeutic antibodies. Semin. Oncol., 37: 508-516.

Baldo, B.A., 2013. Adverse events to monoclonal antibodies used for cancer therapy: Focus on hypersensitivity responses. Oncoimmunology, 2: 1-16.

Burkly, L.C., J.S. Michaelson and T.S. Zheng, 2011. TWEAK/Fn14 pathway: An immunological switch for shaping tissue responses. Immunol. Rev., 244: 99-114.

Camidge, D.R., R.S. Herbst, M.S. Gordon, S.G. Eckhardt and R. Kurzrock et al., 2010. A phase I safety and pharmacokinetic study of the death receptor 5 agonistic antibody PRO95780 in patients with advanced malignancies. Clin. Cancer Res., 16: 1256-1263.

Campbell, S., J. Michaelson, L. Burkly and C. Putterman, 2004. The role of TWEAK/Fn14 in the pathogenesis of inflammation and systemic autoimmunity. Front Biosci., 9: 2273-2284.

Chao, D.T., M. Su, S. Tanlimco, M. Sho and D. Choi et al., 2013. Expression of TweakR in breast cancer and preclinical activity of enavatuzumab, a humanized anti-TweakR mAb. J. Cancer Res. Clin. Oncol., 139: 315-325.

Chodorge, M., S. Zuger, C. Stirnimann, C. Briand and L. Jermutus et al., 2012. A series of Fas receptor agonist antibodies that demonstrate an inverse correlation between affinity and potency. Cell Death Differ, 19: 1187-1195.

Chuntharapai, A., K. Dodge, K. Grimmer, K. Schroeder and S.A. Marsters et al., 2001. Isotype-dependent inhibition of tumor growth in vivo by monoclonal antibodies to death receptor 4. J. Immunol., 166: 4891-4898.

Croft, M., C.A. Benedict and C.F. Ware, 2013. Clinical targeting of the TNF and TNFR super families. Nat. Rev. Drug Discov., 12: 147-168.

Culp, P.A., D. Choi, Y. Zhang, J. Yin and P. Seto et al., 2010. Antibodies to TWEAK receptor inhibit human tumor growth through dual mechanisms. Clin. Cancer Res., 16: 497-508.

Dharmapatni, A.A., M.D. Smith, T.N. Crotti, C.A. Holding and C. Vincent et al., 2011. TWEAK and Fn14 expression in the pathogenesis of joint inflammation and bone erosion in rheumatoid arthritis. Arthritis Res. Ther., 13: 1-10.

Dhein, J., P.T. Daniel, B.C. Trauth, A. Oehm and P. Moller et al., 1992. Induction of apoptosis by monoclonal antibody anti-APO-1 class switch variants is dependent on cross-linking of APO-1 cell surface antigens. J. Immunol., 149: 3166-3173.

Drobyski, W.R., M. Gottlieb, D. Carrigan, L. Ostberg and M. Grebenau et al., 1991. Phase I study of safety and pharmacokinetics of a human anticytomegalovirus monoclonal antibody in allogeneic bone marrow transplant recipients. Transplantation, 51: 1190-1196. 
Field, K.M. and M. Michael, 2008. Part II: Liver function in oncology: Towards safer chemotherapy use. Lancet Oncol, 9: 1181-1190. DOI: $10.1016 / \mathrm{S} 1470-2045(08) 70307-3$

Field, K.M., C. Dow and M. Michael, 2008. Part I: Liver function in oncology: Biochemistry and beyond. Lancet Oncol., 9: 1092-1101. DOI: 10.1016/S1470-2045(08)70279-1

Galle, P.R., W.J. Hofmann, H. Walczak, H. Schaller and G. Otto et al., 1995. Involvement of the CD95 (APO-1/Fas) receptor and ligand in liver damage. J. Exp. Med., 182: 1223-1230.

Giavedoni, L.D., 2005. Simultaneous detection of multiple cytokines and chemokines from nonhuman primates using luminex technology. J. Immunol. Methods, 301: 89-101.

Gomez-Lechon, M.J., A. Lahoz, L. Gombau, J.V. Castell and M.T. Donato, 2010. In vitro evaluation of potential hepatotoxicity induced by drugs. Curr. Pharm. Des., 16: 1963-1977.

Jakubowski, A., C. Ambrose, M. Parr, J.M. Lincecum and M.Z. Wang et al., 2005. TWEAK induces liver progenitor cell proliferation. J. Clin. Invest, 115: 2330-2340.

Kaplowitz, N., 2004. Drug-induced liver injury. Clin. Infect. Dis., 38: S44-S48. DOI: 10.1086/381446

Kimura, K., H. Moriwaki, M. Nagaki, M. Saio and Y. Nakamoto et al., 2006. Pathogenic role of B cells in anti-CD40-induced necroinflammatory liver disease. Am. J. Pathol., 168: 786-795.

Lake, B.G. and R.J. Price, 2013. Evaluation of the metabolism and hepatotoxicity of xenobiotics utilizing precision-cut slices. Xenobiotica, 43: 41-53.

Lam, E.T., S.G. Eckhardt, W. Messersmith, A. Jimeno and C.L. O'Bryant et al., 2017. Phase I study of enavatuzumab, a first-in-class humanized monoclonal antibody targeting the TWEAK (tumor necrosis factor-like inducer of apoptosis) receptor, in patients with advanced solid tumors. Molecular Cancer Therapeutics.

Leifeld, L., C. Trautwein, F.L. Dumoulin, M.P. Manns and T. Sauerbruch et al., 1999. Enhanced expression of CD80 (B7-1), CD86 (B7-2) and CD40 and their ligands CD28 and CD154 in fulminant hepatic failure. Am. J. Pathol., 154: 1711-1720.

Li, B., S.J. Russell, D.M. Compaan, K. Totpal and S.A. Marsters et al., 2006. Activation of the proapoptotic death receptor DR5 by oligomeric peptide and antibody agonists. J. Mol. Biol., 361: 522-536.

Lu, J., B.C. Kwan, F.M. Lai, P.C. Choi and L.S. Tam et al., 2011. Gene expression of TWEAK/Fn14 and IP10/CXCR3 in glomerulus and tubulointerstitium of patients with lupus nephritis. Nephrology, 16: 426-432.
Napetschnig, J. and H. Wu, 2013. Molecular basis of NFkappaB signaling. Ann. Rev. Biophys., 42: 443-468.

Niu, L., S. Strahotin, B. Hewes, B. Zhang and Y. Zhang et al., 2007. Cytokine-mediated disruption of lymphocyte trafficking, hemopoiesis and induction of lymphopenia, anemia and thrombocytopenia in anti-CD137-treated mice. J. Immunol., 178: 4194-4213.

Ogasawara, J., R. Watanabe-Fukunaga, M. Adachi, A. Matsuzawa and T. Kasugai et al., 1993. Lethal effect of the anti-Fas antibody in mice. Nature, 364: 806-809.

Plummer, R., G. Attard, S. Pacey, L. Li and A. Razak et al., 2007. Phase 1 and pharmacokinetic study of lexatumumab in patients with advanced cancers. Clin. Cancer Res., 13: 6187-6194.

Purcell, J.W., H.K. Kim, S.G. Tanlimco, M. Doan and M. Fox et al., 2014. Nuclear factor $\kappa \mathrm{B}$ is required for tumor growth inhibition mediated by enavatuzumab (PDL192), a humanized monoclonal antibody to TweakR. Front Immunol., 4: 1-13. DOI: $10.3389 /$ fimmu.2013.00505

Saez, M.I., C. Quero, J.M. Trigo, B. Muros and E. Alba, 2012. Practical guidelines for dose individualization of anticancer targeted drugs. Clin. Transl. Oncol., 14: 812-819.

Sahi, J., S. Grepper and C. Smith, 2010. Hepatocytes as a tool in drug metabolism, transport and safety evaluations in drug discovery. Curr. Drug Discov. Technol., 7: 188-198.

Salzmann, S., A. Seher, J. Trebing, D. Weisenberger and A. Rosenthal et al., 2013. Fibroblast growth factor inducible (Fn14)-specific antibodies concomitantly display signaling pathway-specific agonistic and antagonistic activity. J. Biol. Chem., 288: 13455-13466.

Schwabe, R.F., B. Schnabl, Y.O. Kweon and D.A. Brenner, 2001. CD40 activates NF-kappa B and c-Jun Nterminal kinase and enhances chemokine secretion on activated human hepatic stellate cells. J. Immunol., 166: 6812-6819.

Serafini, B., R. Magliozzi, B. Rosicarelli, R. Reynolds and T.S. Zheng et al., 2008. Expression of TWEAK and its receptor Fn14 in the multiple sclerosis brain: implications for inflammatory tissue injury. J. Neuropathol. Exp. Neurol., 67: 1137-1148.

Soldatow, V.Y., E.L. Lecluyse, L.G. Griffith and I. Rusyn, 2013. In vitro models for liver toxicity testing. Toxicol. Res., 2: 23-39.

Tolcher, A.W., M. Mita, N.J. Meropol, M. von Mehren and A. Patnaik et al., 2007. Phase I pharmacokinetic and biologic correlative study of mapatumumab, a fully human monoclonal antibody with agonist activity to tumor necrosis factor-related apoptosis-inducing ligand receptor-1. J. Clin. Oncol., 25: 1390-1395. 
van Kuijk, A.W.R., C.A. Wijbrandts, M. Vinkenoog, T.S. Zheng and K.A. Reedquist et al., 2010. TWEAK and its receptor Fn14 in the synovium of patients with rheumatoid arthritis compared to psoriatic arthritis and its response to tumour necrosis factor blockade. Ann. Rheum. Dis., 69: 301-304.

Volkmann, X., U. Fischer, M.J. Bahr, M. Ott and F. Lehner et al., 2007. Increased hepatotoxicity of tumor necrosis factor-related apoptosis-inducing ligand in diseased human liver. Hepatology, 46: 1498-1508.

Vonderheide, R.H., K.T. Flaherty, M. Khalil, M.S. Stumacher and D.L. Bajor et al., 2007. Clinical activity and immune modulation in cancer patients treated with CP-870,893, a novel CD40 agonist monoclonal antibody. J. Clin. Oncol., 25: 876-883.
White, A.L, H.T. Chan, R.R. French, S.A. Beers and M.S. Cragg et al., 2013. Fc $\gamma$ RIIB controls the potency of agonistic anti-TNFR mAbs. Cancer Immunol. Immunother, 62: 941-948.

Wiley, S.R., L. Cassiano, T. Lofton, T. Davis-Smith and J.A. Winkles et al., 2001. A novel TNF receptor family member binds TWEAK and is implicated in angiogenesis. Immunity, 15: 837-846.

Younes, A. and M.E. Kadin, 2003. Emerging applications of the tumor necrosis factor family of ligands and receptors in cancer therapy. J. Clin. Oncol., 21: 3526-3534.

Zheng, T.S. and L.C. Burkly, 2008. No end in site: TWEAK/Fn14 activation and autoimmunity associated-end-organ pathologies. J. Leukoc Biol., 84: 338-347.

Zimmerman, H.J., 2000. Drug-induced liver disease. Clin. Liver Dis., 4: 73-96.

DOI: $10.1016 / \mathrm{S} 1089-3261(05) 70097-0$ 